\title{
Utilization of Mental Healthcare Services among Refugees: Past, Present and Future
}

Huaibo Xin

Department of Applied Health, Southern Illinois University Edwardsville, Campus Box 1126, SIUE, Edwardsville, IL62026, USA.

\author{
Article Details \\ Article Type: Research Article \\ Received date: $18^{\text {th }}$ December, 2017 \\ Accepted date: $19^{\text {th }}$ March, 2018 \\ Published date: $26^{\text {th }}$ March, 2018
}

"Corresponding Author: Huaibo Xin, Department of Applied Health, Southern Illinois University Edwardsville Campus Box 1126, SIUE, Edwardsville, IL62026, USA. E-mail. hxin@siue.edu

Citation: Xin H (2018) Utilization of Mental Healthcare Services among Refugees: Past, Present and Future. J Pub Health Issue Pract 2: 112. https://doi.org/10.33790/jphip1100112.

Copyright: ( 2 2018, This is an open-access article distributed under the terms of the Creative Commons Attribution License 4.0, which permits unrestricted use, distribution, and reproduction in any medium, provided the original author and source are credited.

\begin{abstract}
There has been an increasingly large number of refugees in the U.S. and worldwide. Compared to the general population, historically, refugees presented a higher prevalence and incidence of mental disorders due to their traumatic/stressful experiences. However, the unmet needs of mental healthcare among refugees remain significant. The purpose of this study is to systematically review the current literature and identify the challenges that refugees were facing to receive mental healthcare in western countries as well as clinical and non-clinical ap- proaches that may effectively address their mental healthcare needs. A total of 79 peer-reviewed journal articles, reports, and book chapters published between 1985 and 2018 and obtained from major databases were included in the final analysis. Data were extracted and synthesized into categories of barriers for refugees to utilizing mental healthcare in the U.S. and other western countries, and effectiveness of current approaches to address refugees' mental illnesses. Results indicated that the vast majority of barriers existed in both U.S. and other western countries were common. In response, four themes emerged to improve refugees' utilization of mental healthcare, including adopting effective westernized trauma-focused mental health treatment; recognizing traditional and culturally relevant mental health coping mechanism; overcoming existing practical barriers within both refugee communities and western mental healthcare systems; emphasizing comprehensive/integrative mental healthcare models. Future endeavor should focus more on systematically integrating planned cultural competency training and education into existing curriculum, strengthening the quality of mental healthcare research with refugees, and advocating for funding support for mental healthcare services, training, and research.
\end{abstract}

Key Words: Mental Healthcare, Refugees, Utilizations, Barriers, Solutions

\section{Introduction}

According to the United Nation Refugee Agency (UNHCR) (2018), currently, worldwide, approximately 65.6 million people have been forcibly displaced and hosted by countries or districts in Africa, Middle East and North Africa, Europe, Americas, and Asia and Pacific, which raised the annual financial burden up to 1.1 billion dollars in 2018 [1]. Among those, about 22.5 million people are refugees, and $55 \%$ of the refugees fled from countries, including Syria, Afghanistan, and South Sudan. More than 11 million refugees are under the age of 18 . In the year of 2016, a total of 189,300 forcibly became refugees [1]. In the United States, since 1975, more than 3 million refugees have been admitted and resettled throughout the country with about $40 \%$ being aged 17 years old or younger [2]. U.S. refugees came from countries in Africa, Europe, Latin America and Caribbean, and Near East and South Asia. From October 2016 to August 2018, over 39\% refugees were from countries in near East and South Asia, such as Iraq, Syria, Bhutan, and Iran; over 37\% refugees were from African countries, including democratic republic of Congo, Somalia, and Eritrea; and about 19\% was from Asian and European countries like Burma and Ukraine [2]. The origin of the waves of the refugee population coming to U.S. has shifted from Asia, former Soviet Union, to near East and South Asia over the past 33 years. California, Texas, New York, Ohio, and Michigan currently are the states hosted most of the refugees. As of July 30, in the fiscal year of 2018, 5,0479 refugees have already been admitted to the U.S. compared to a total of 8,4994 admissions in the fiscal year of 2016 [2].

Historically, compared to the general population, refugee population has presented a higher risk of suffering from mental disorders, including anxiety, depression, and/or post-traumatic stress disorder (PTSD) [3-7]. Results from 6,743 adult refugees from seven countries involved in 20 surveys between 1966 and 2002 were systematically reviewed. All refugees were resettled in western countries with more than $69 \%$ of the study participants were living in the U.S. PTSD was more significant than depression and anxiety, which indicated it could be ten times more prevalent among refugees than it was among age-matched general population in their resettlement countries [4]. A meta-analysis and systematic review further looked into the prevalence of depression and anxiety among both refugees and labor immigrants.

A total of 35 studies between 1990 and 2007 were chosen, and 24,051 multiethnic subjects immigrated to ei- ther eastern or western countries were included. A very majority of the subjects were adults, and about $62 \%$ of them immigrated to the U.S. Compared to the labor immigrants, refugees had twice higher prevalence rate of both depression and anxiety [5]. Respectively, 366 Hopkins Symptom Checklists were completed and received from 366 Iraqi refugee adults residing in Georgia, Michigan, California, and Texas. Approximately, $50 \%$ of the participants reported symptoms of 
depression, anxiety, and emotional distress, and $31 \%$ were at risk of PTSD [8]. Studies on Afghanistan refugees resettled in countries, such as U.S., Australia, Germany, UK, Japan, and Netherlands, found out that the prevalence rate of depression, anxiety, and PTSD were significantly high. Mental health measurements, including Hopkins Symptom Checklist, Beck Depression Inventory, and Center for Epidemiologic Studies Depression Scale, were adopted by these studies $[3,9]$. Especially with Afghanistan refugee women, one study showed a much poorer mental health status among 423 women respondents than it was among 272 male respondents, and a statistically significantly higher prevalence rate in depression, anxiety, or PTSD among Afghanistan women [9]. Six studies examining more than 4,700 Bhutanese refugees' mental health also indicated the predominant mental disorders as well as torture experiences [6]. Very similar results were found among many other refugee adult populations, including Iranians, Cambodians, Somali, and Hmongs, living all over the world [10-12]. These psychiatric symptoms can be carried over for decades due to the perseverant impact of premigration, migration, and post-migration traumas [12, 13].

Psychiatric distress is also prevalent among refugee children under the age of 18 [14-17]. In a comparative study, 101 refugee children in the age range of 5 to 18 were compared with age and gender matched 101 ethnic minority children and 101 white children living in U.K. The Strengths and Difficulties Questionnaire was used to measure the children's mental distress. Respectively, refugee children were about 3 times more likely and 2 times more likely to develop psychiatric symptoms than ethnic minorities and Whites [16]. A total of 135 Somali refugee adolescents living in the U.S. responded to the UCLA PTSD Index and Depression Self-Rating Scale. A significantly high prevalence of both PTSD and depression were predicted among the participants, which was associated with their pre-resettlement accumulative trauma experiences and perceived discrimination and stress in their host country [15]. Recent systematic reviews of studies on children or youth's mental health suggested the consistency of previous findings that indicated the significance of minors' both susceptibility and severity of psychiatric disorders [14, 17]. For instance, a meta-analysis was conducted among 7,920 refugee children aged between 5 and 17 from 17 studies published between 1989 and 2007. The overall corresponding estimate of prevalence of PTSD, depression, and anxiety was $47 \%, 43 \%$, and $27 \%$.

Elevated occurrence of PTSD was also identified among Iran, Iraq, Israel, Kuwait, and Palestine refugee mi- nors [14]. Evidence has further demonstrated the unmet needs of addressing refugee populations' mental health and demonstrated both disease and financial burden caused by their mental disorders [18-23]. Through a system- atic review, this study is to synthesize the major barriers to the utilization of mental healthcare services among refugee populations and examine the effectiveness of current clinical and non-clinical approaches to address refugees' mental healthcare needs.

\section{Methods}

\section{Inclusion and Exclusion Criteria}

Peer-reviewed journal articles, reports, and books related to refugee adults' and adolescents', immigrants', and asylum seekers' mental healthcare published between 1985 and 2018 and using qualitative, quantitative, or mixed research methods, systematic review, or metaanalysis were reviewed. Articles clearly discussing either barriers to utilizing mental healthcare and/or effectiveness of mental healthcare strategies among different international refugee populations resettled in western countries were reviewed. Both clinical and non-clinical mental healthcare approaches and coping mechanism adopted by either clinicians or refugees themselves to manage symptoms of PTSD, depression, anxiety, and other mental distress, which explicitly demonstrated the effectiveness of mental healthcare strategies, were reviewed. Articles only discussing mental healthcare among asylum seekers or immigrants were excluded from the current study.

\section{Search Strategy}

A combination of "mental healthcare and refugees" was used as search words. Databases, including PubMed, Medline, Medline Plus, PsycINFO, EBSCO, and CINAHL, as well as Google Scholar were independently used to extensively search for the relevant peerreviewed journal articles, reports, and book chapters. A total of 1,058 peer-reviewed journal articles, reports, and book chapters were initially reviewed, and 67 articles relevant to the effectiveness of both clinical and non-clinical approaches to alleviate the severity of refugees' mental distress and 93 articles relevant to the barriers to utilizing mental healthcare among refugees in their host countries were selected for a further review. Only 79 peer-reviewed journal articles, reports, and book chapters were included in the final analysis. Among those, 32 articles explicitly discussed the barriers to utilizing mental healthcare ser- vices among refugees resettled in western countries, 47 articles illustrated the effectiveness of mental healthcare approaches, and 8 articles discussed both.

\section{Data Extraction and Synthesis}

Articles concerning refugee populations' challenges of receiving mental healthcare were reviewed. Information was extracted based on the areas in refugees' help-seeking behavior, refugees' perceptions of mental healthcare, and availability, accessibility, and affordability of mental healthcare, which hindered refugees' utilization of mental healthcare in a developed country. Information was then synthesized using theme analysis, and comparisons of barriers to utilizing mental healthcare were made between U.S. and other western countries. Articles indicating the effectiveness of a variety of mental healthcare approaches among refugees in western countries were also examined to identify the unique characteristics of effective clinical and nonclinical strategies, which were tailored to the refugees' care, and were then compiled by theme.

\section{Results}

Table 1 presents the existing barriers to utilizing mental healthcare services among multiethnic refugee populations resettled in the U.S. [24-55]. Refugee populations hesitated to seek western mental healthcare was often because of a lack of knowledge and understanding of the westernized definition of mental health, the impact of traumas on their mental wellbeing, as well as the westernized terminologies of mental disorders and interpretation of symptoms [24-34]. They culturally interpreted that the causes of mental disorders were factors, such as "socio-environmental stressors" instead of internal or biological factors, and spirituality, and culturally explained their symptoms using physical discomforts [28-29, 30, 32, 33]. They believed mild psychiatric symptoms can be absorbed by talking to their family members [28]. There was also a lack of support from refugee patients' family and community for them to seek professional mental healthcare $[26,28,35]$. In addition, refugees were not used to scheduling an appointment for their medical needs, having a long wait for their appointment, and following up with multiple counseling sessions for treatment, which was very different from the care they received in their home countries [24-30]. They also had a hard time navigating mental healthcare services in their host country and lacked awareness of their availability [24, 32, 37]. They hesitated to initiate a conversation with their mental healthcare providers about their past traumatic experiences due to their silent culture, mistrust of authorities, and concerns of confidentiality and privacy $[25,26$, $29,33,34,38]$. They hesitated to fully disclose their psychiatric symptoms because of their concerns about stigmas of having mental disorders and fears of being separated from their families if being hospitalized [25, 27, 28, 31, 32, 34, 37, 38]. Some refugee groups also expressed concerns about the high medical costs, unacceptability of pharmaceuticals, and inconvenience of transportation to a care facility $[27,28,37]$. 


\begin{tabular}{|c|c|c|c|c|c|}
\hline Relevance & $\begin{array}{l}\text { Barriers to Mental } \\
\text { Healthcare }\end{array}$ & $\begin{array}{l}\text { Target Refugee } \\
\text { Population }\end{array}$ & $\begin{array}{l}\text { Study } \\
\text { Population Age }\end{array}$ & Research Design & $\begin{array}{l}\text { Authors and Year of } \\
\text { Publication }\end{array}$ \\
\hline \multirow[t]{3}{*}{ Refugee Patients } & $\begin{array}{l}\text { Lack of } \\
\text { understanding of } \\
\text { the process and/ } \\
\text { or terminologies } \\
\text { of western mental } \\
\text { health treatment }\end{array}$ & $\begin{array}{l}\text { 1. Multiple } \\
\text { countries } \\
\text { 2. Vietnam } \\
\text { 3. Asia } \\
\text { 4. Cambodia } \\
\text { 5. Somali } \\
\text { 6. Somali } \\
\text { 7. Southeast Asia }\end{array}$ & $\begin{array}{l}\text { 1. Adults } \\
\text { 2. Not specified } \\
\text { 3. Not specified } \\
\text { 4. Adults } \\
\text { 5. Adults } \\
\text { 6. Adults } \\
\text { 7. Adults }\end{array}$ & $\begin{array}{l}\text { 1. S y s t e m a t i c } \\
\text { review } \\
\text { 2. Commentary } \\
\text { 3. Review } \\
\text { 4. Primary research } \\
\text { 5. Primary research } \\
\text { 6. Primary research } \\
\text { 7. Primary research }\end{array}$ & $\begin{array}{l}\text { 1. Gong-Guy E., Cravens } \\
\text { RB., \& Patterson TE. (1991) } \\
\text { 2. Gold SJ. (1992) } \\
\text { 3. Dhooper SS., \& Tran } \\
\text { TV. (1998) } \\
\text { 4. Blair RG. (2001) } \\
\text { 5. Scuglik et al. (2007) } \\
\text { 6. Pavlish CL., Noor S., } \\
\text { \& Brandt J. (2010) } \\
\text { 7. Wagner et al. (2013) }\end{array}$ \\
\hline & $\begin{array}{l}\text { Stigma of mental } \\
\text { disorders }\end{array}$ & $\begin{array}{l}\text { 1. Vietnam } \\
\text { 2. Cambodia } \\
\text { 3. Cambodia } \\
\text { 4. Somali } \\
\text { 5. Multiple } \\
\text { countries } \\
\text { 6. Southeast } \\
\text { Asia } \\
\text { 7. Multiple } \\
\text { countries }\end{array}$ & $\begin{array}{l}\text { 1. Not specified } \\
\text { 2. Adolescents } \\
\text { 3. Adults } \\
\text { 4. Adults } \\
\text { 5. Adults } \\
\text { 6. Adults } \\
\text { 7. Adults }\end{array}$ & $\begin{array}{l}\text { 1. Commentary } \\
\text { 2. Commentary } \\
\text { 3. Primary research } \\
\text { 4. Primary research } \\
\text { 5. Primary research } \\
\text { 6. Primary research } \\
\text { 7. Primary research }\end{array}$ & $\begin{array}{l}\text { 1. Gold SJ. (1992) } \\
\text { 2. Frye BA., \& McGill } \\
\text { D. (1993) } \\
\text { 3. Blair RG. (1998) } \\
\text { 4. Scuglik et al. (2007) } \\
\text { 5. Morris et al. (2009) } \\
\text { 6. Lee HY. Lytle K., } \\
\text { Yang PN., \& Lum T (2010) } \\
\text { 7. Shannon PJ., Wieling } \\
\text { E., Simelink-Mccleary } \\
\text { J., \& Becher E. (2015) }\end{array}$ \\
\hline & $\begin{array}{l}\text { Culturally oriented } \\
\text { health beliefs and } \\
\text { interpretation } \\
\text { of the causes of } \\
\text { mental illnesses }\end{array}$ & $\begin{array}{l}\text { 1. Southeast Asia } \\
\text { 2. Somali } \\
\text { 3. Somali } \\
\text { 4. Southeast Asia } \\
\text { 5. Somali } \\
\text { 6. M u } 1 \mathrm{t} \mathrm{i} \mathrm{p} 1 \text { e } \\
\text { countries } \\
\text { 7. Southeast Asia }\end{array}$ & $\begin{array}{l}\text { 1. Adults and } \\
\text { children } \\
\text { 2. Adults } \\
\text { 3. Adults } \\
\text { 4. Adults } \\
\text { 5. Adults } \\
\text { 6. Adults } \\
\text { 7. Adults }\end{array}$ & $\begin{array}{l}\text { 1. Primary research } \\
\text { 2. Primary research } \\
\text { 3. Primary research } \\
\text { 4. Primary research } \\
\text { 5. Primary research } \\
\text { 6. Primary research } \\
\text { 7. Primary research }\end{array}$ & $\begin{array}{l}\text { 1. Nguyen SD. (1985) } \\
\text { 2. Carroll JK. (2004) } \\
\text { 3. Scuglik et al. (2007) } \\
\text { 4. Lee HY. Lytle K., } \\
\text { Yang PN., \& Lum T (2009) } \\
\text { 5. Pavlish CL., Noor S., } \\
\text { \& Brandt J. (2010) } \\
\text { 6. Shannon P., } \\
\text { O’Dougherty M., \& Mehta E. } \\
\text { (2012) } \\
\text { 7. Wagner et al. (2013) }\end{array}$ \\
\hline & $\begin{array}{l}\text { Lack of knowledge } \\
\text { of mental disorders } \\
\text { and the impact of } \\
\text { traumas on mental } \\
\text { wellbeing. }\end{array}$ & $\begin{array}{l}\text { 1. Vietnam } \\
\text { 2. Asia } \\
\text { 3. Multiple } \\
\text { countries } \\
\text { 4. Southeast } \\
\text { Asia } \\
\text { 5. Multiple } \\
\text { Countries } \\
\text { 6. Multiple } \\
\text { countries }\end{array}$ & $\begin{array}{l}\text { 1. Not specified } \\
\text { 2. Not specified } \\
\text { 3. Adults } \\
\text { 4. Adults } \\
\text { 5. Adults } \\
\text { 6. Adults }\end{array}$ & $\begin{array}{l}\text { 1. Commentary } \\
\text { 2. Review } \\
\text { 3. Primary research } \\
\text { 4. Primary research } \\
\text { 5. Primary research } \\
\text { 6. Primary research }\end{array}$ & $\begin{array}{l}\text { 1. Gold SJ. (1992) } \\
\text { 2. Dhooper SS., \& Tran } \\
\text { TV. (1998) } \\
\text { 3. Morris et al. (2009) } \\
\text { 4. Lee HY. Lytle K., Yang } \\
\text { PN., \& Lum T. } \\
\text { (2010) } \\
\text { 5. Shannon P., O'Dougherty } \\
\text { M., \& Mehta E. (2012) } \\
\text { 6. Shannon PJ.,Wieling E., } \\
\text { Simelink-Mccleary J., \& } \\
\text { Becher E. (2015) }\end{array}$ \\
\hline & $\begin{array}{l}\text { Lack of an } \\
\text { initiative of a } \\
\text { conversation } \\
\text { with service } \\
\text { providers about } \\
\text { traumatic } \\
\text { experiences. }\end{array}$ & $\begin{array}{l}\text { 1. Vietnam } \\
\text { 2. Cambodia } \\
\text { 3. Somali } \\
\text { 4. Multiple } \\
\text { countries } \\
\text { 5. Multiple } \\
\text { countries }\end{array}$ & $\begin{array}{l}\text { 1. Not specified } \\
\text { 2. Adolescents } \\
\text { 3. Adults } \\
\text { 4. Adults } \\
\text { 5. Adults }\end{array}$ & $\begin{array}{l}\text { 1. Commentary } \\
\text { 2. Commentary } \\
\text { 3. Primary research } \\
\text { 4. Primary research } \\
\text { 5. Primary research }\end{array}$ & $\begin{array}{l}\text { 1. Gold SJ. (1992) } \\
\text { 2. Frye BA., \& McGill D. } \\
\text { (1993) } \\
\text { 3. Pavlish CL., Noor S., \& } \\
\text { Brandt J. (2010) } \\
\text { 4. Shannon P., O’Dougherty } \\
\text { M., \& Mehta E. (2012) } \\
\text { 5. Shannon PJ., Wieling E., } \\
\text { Simelink-Mccleary J., } \\
\text { \& Becher E. (2015) }\end{array}$ \\
\hline
\end{tabular}

Table 1. to be Cont.... 


\begin{tabular}{|c|c|c|c|c|c|}
\hline & Language barriers & $\begin{array}{l}\text { 1. Cambodia } \\
\text { 2. Asia } \\
\text { 3. Somali } \\
\text { 4. Somali }\end{array}$ & $\begin{array}{l}\text { 1. Adolescents } \\
\text { 2. Not specified } \\
\text { 3. Adults } \\
\text { 4. Adults }\end{array}$ & $\begin{array}{l}\text { 1. Commentary } \\
\text { 2. Review } \\
\text { 3. Primary research } \\
\text { 4. Primary research }\end{array}$ & $\begin{array}{l}\text { 1. Frye BA., \& McGill } \\
\text { D. (1993) } \\
\text { 2. Dhooper SS., \& Tran } \\
\text { TV. (1998) } \\
\text { 3. Scuglik et al. (2007) } \\
\text { 4. Bettmann JE., Penney } \\
\text { D., Freeman PC., \& } \\
\text { Lecy N. (2015) }\end{array}$ \\
\hline & $\begin{array}{l}\text { Lack of social support } \\
\text { of utilization of mental } \\
\text { healthcare services }\end{array}$ & $\begin{array}{l}\text { 1. Asia } \\
\text { 2. Somali } \\
\text { 3. Somali }\end{array}$ & $\begin{array}{l}\text { 1. Not specified } \\
\text { 2. Adults } \\
\text { 3. Adults }\end{array}$ & $\begin{array}{l}\text { 1. Review } \\
\text { 2. Primary research } \\
\text { 3. Primary research }\end{array}$ & $\begin{array}{l}\text { 1. Dhooper SS., \& Tran } \\
\text { TV. (1998) } \\
\text { 2. Scuglik et al. (2007) } \\
\text { 3. Bettmann JE., Penney } \\
\text { D., Freeman PC., \& } \\
\text { Lecy N. (2015) }\end{array}$ \\
\hline & $\begin{array}{l}\text { Lack of trust of } \\
\text { authorities }\end{array}$ & $\begin{array}{l}\text { 1.Vietnam } \\
\text { 2. Asia }\end{array}$ & $\begin{array}{l}\text { 1. Not specified } \\
\text { 2. Not specified }\end{array}$ & $\begin{array}{l}\text { 1. Commentary } \\
\text { 2. Review }\end{array}$ & $\begin{array}{l}\text { 1. Gold SJ. (1992) } \\
\text { 2. Dhooper SS., \& Tran } \\
\text { TV. (1998) }\end{array}$ \\
\hline & $\begin{array}{l}\text { Concerns about medical } \\
\text { cost }\end{array}$ & $\begin{array}{l}\text { 1. Somali } \\
\text { 2. Southeast Asia }\end{array}$ & $\begin{array}{l}\text { 1. Adults } \\
\text { 2. Adults }\end{array}$ & $\begin{array}{l}\text { 1. Primary research } \\
\text { 2. Primary research }\end{array}$ & $\begin{array}{l}\text { 1. Scuglik et al. (2007) } \\
\text { 2. Wagner et al. (2013) }\end{array}$ \\
\hline & $\begin{array}{l}\text { Lack of acceptability of } \\
\text { pharmaceuticals }\end{array}$ & 1. Somali & 1. Adults & 1. Primary research & $\begin{array}{l}\text { 1. Bettmann JE., Penney } \\
\text { D., Freeman PC., \& } \\
\text { Lecy N. (2015) }\end{array}$ \\
\hline & $\begin{array}{l}\text { Fear of being separated } \\
\text { from family due to } \\
\text { hospitalization }\end{array}$ & 1. Somali & 1. Adults & 1. Primary research & $\begin{array}{l}\text { 1. Bettmann JE., Penney } \\
\text { D., Freeman PC., \& } \\
\text { Lecy N. (2015) }\end{array}$ \\
\hline & $\begin{array}{l}\text { Inconvenience } \\
\text { transportation }\end{array}$ & 1. Asia & 1. Adults & 1. Primary research & 1. Blair RG. (2001) \\
\hline \multirow[t]{3}{*}{$\begin{array}{l}\text { Mental } \\
\text { Healthcare } \\
\text { System }\end{array}$} & $\begin{array}{l}\text { Lack of professional } \\
\text { interpreters and/ } \\
\text { or translators }\end{array}$ & $\begin{array}{l}\text { 1. Multiple } \\
\text { countries } \\
\text { 2. Cambodia } \\
\text { 3. Asia } \\
\text { 4. Cambodia } \\
\text { 5. Southeast Asia } \\
\text { 6. Southeast Asia }\end{array}$ & $\begin{array}{l}\text { 1. Adults and } \\
\text { children } \\
\text { 2. Adolescents } \\
\text { 3. Not specified } \\
\text { 4. Adults } \\
\text { 5. Adults } \\
\text { 6. Adults }\end{array}$ & $\begin{array}{l}\text { 1. Systematic review } \\
\text { 2. Commentary } \\
\text { 3. Review } \\
\text { 4. Primary research } \\
\text { 5. Primary research } \\
\text { 6. Primary research }\end{array}$ & $\begin{array}{l}\text { 1.Gong-Guy E., } \\
\text { Cravens } \\
\text { RB., \& Patterson TE. } \\
\text { (1991) } \\
\text { 2. Frye BA., \& McGill } \\
\text { D.(1993) } \\
\text { 3. Dhooper SS., \& Tran } \\
\text { TV. (1998) } \\
\text { 4. Blair RG.(2001) } \\
\text { 5. Lee HY. Lytle K., } \\
\text { Yang PN., \& Lum T } \\
\text { (2010) } \\
\text { 6. Wagner et al. (2013) }\end{array}$ \\
\hline & $\begin{array}{l}\text { Lack of culturally } \\
\text { and linguistic competent } \\
\text { mental healthcare } \\
\text { providers }\end{array}$ & $\begin{array}{l}\text { 1. Multiple } \\
\text { countries } \\
\text { 2. Vietnam } \\
\text { 3. Asia } \\
\text { 4. Cambodia }\end{array}$ & $\begin{array}{l}\text { 1. Adults and } \\
\text { children } \\
\text { 2. Not specified } \\
\text { 3. Not specified } \\
\text { 4. Adults }\end{array}$ & $\begin{array}{l}\text { 1. Systematic } \\
\text { review } \\
\text { 2. Commentary } \\
\text { 3. Review } \\
\text { 4. Primary research }\end{array}$ & $\begin{array}{l}\text { 1. Gong-Guy E., } \\
\text { Cravens RB., \& } \\
\text { Patterson TE. (1991) } \\
\text { 2. Gold SJ. (1992) } \\
\text { 3. Dhooper SS., \& Tran } \\
\text { TV. (1998) } \\
\text { 4. Blair RG. (2001) }\end{array}$ \\
\hline & $\begin{array}{l}\text { Lack of tailored mental } \\
\text { health services for } \\
\text { refugees }\end{array}$ & $\begin{array}{l}\text { 1. Southeast Asia } \\
\text { 2. Multiple } \\
\text { countries } \\
\text { 3. Cambodia } \\
\text { 4. Multiple } \\
\text { countries }\end{array}$ & \begin{tabular}{|l|} 
1. Adults and \\
children \\
2. Adults and \\
children \\
3. Adults \\
4. Adults
\end{tabular} & $\begin{array}{l}\text { 1. Primary research } \\
\text { 2. Systematic } \\
\text { review } \\
\text { 3. Primary research } \\
\text { 4. Primary research }\end{array}$ & $\begin{array}{l}\text { 1. Nguyen SD. } \\
\text { (1985) } \\
\text { 2. Gong-Guy E., } \\
\text { Cravens RB., \& } \\
\text { Patterson TE. (1991) } \\
\text { 3. Blair RG. (2001) } \\
\text { 4. Morris et al. (2009) }\end{array}$ \\
\hline
\end{tabular}

Table 1. to be Cont...... 


\begin{tabular}{|l|l|l|l|l|l|}
\hline & $\begin{array}{l}\text { Diagnostic } \\
\text { difficulties due to } \\
\text { lack of } \\
\text { cross-cultural } \\
\text { diagnostic skills }\end{array}$ & $\begin{array}{l}\text { 1. Multiple } \\
\text { countries } \\
\text { 2. Cambodia }\end{array}$ & $\begin{array}{l}\text { 1. Adults and } \\
\text { children } \\
\text { 2. Adolescents }\end{array}$ & $\begin{array}{l}\text { 1. Systematic } \\
\text { review } \\
\text { 2. Commentary }\end{array}$ & $\begin{array}{l}\text { 1. Gong-Guy E., } \\
\text { Cravens RB., \& } \\
\text { Patterson TE. } \\
(1991) \\
\text { 2. Frye BA., \& } \\
\text { McGill D. (1993) }\end{array}$ \\
\hline & $\begin{array}{l}\text { Lack of access to } \\
\text { health insurance }\end{array}$ & 1. Asia & 1. Not specified & 1. Review & $\begin{array}{l}\text { 1. Dhooper SS., \& } \\
\text { Tran TV. (1998) }\end{array}$ \\
\hline & $\begin{array}{l}\text { Inflexibility of } \\
\text { operation hours }\end{array}$ & 1. Cambodia & 1. Adults & 1. Primary research & 1. Blair RG. (2001) \\
\hline
\end{tabular}

Table 1: Barriers to Utilizing Mental Healthcare Services among Refugees Resettled in U.S.

The vast majority of challenges presented in the U.S. concerning refugees' underutilization of mental health- care services were also presented in other high-income western countries, including Canada, Australia, and UK (Table 2) [39-55].

\begin{tabular}{|c|c|c|c|c|c|}
\hline Relevance & $\begin{array}{l}\text { Barriers to Mental } \\
\text { Healthcare }\end{array}$ & $\begin{array}{l}\text { Target Refugee } \\
\text { Population }\end{array}$ & $\begin{array}{l}\text { Study Population } \\
\text { Age }\end{array}$ & Research Design & $\begin{array}{l}\text { Authors and Year of } \\
\text { Publication }\end{array}$ \\
\hline Refugee Patients & $\begin{array}{l}\text { Stigma of mental } \\
\text { disorders }\end{array}$ & $\begin{array}{l}\text { 1. Multiple } \\
\text { countries } \\
\text { 2. Multiple } \\
\text { countries } \\
\text { 3. Somali } \\
\text { 4. Somali } \\
\text { 5. Multiple } \\
\text { countries } \\
\text { 6. Multiple } \\
\text { countries } \\
\text { 7. Multiple } \\
\text { countries } \\
\text { 8. Iraq }\end{array}$ & $\begin{array}{l}\text { 1. Adults } \\
\text { 2. Adults } \\
\text { 3. Adults } \\
\text { 4. Adults } \\
\text { 5. Adults } \\
\text { 6. Adults } \\
\text { 7. Adolescents } \\
\text { 8. Adults }\end{array}$ & $\begin{array}{l}\text { 1. Commentary } \\
\text { 2. Primary research } \\
\text { 3. Primary research } \\
\text { 4. Primary research } \\
\text { 5. Primary research } \\
\text { 6. Primary research } \\
\text { 7. Primary research } \\
\text { 8. Primary research }\end{array}$ & $\begin{array}{l}\text { 1. Murphy et al. (2002) } \\
\text { 2. Misra T., Connolly } \\
\text { AM., \& Majeed A. } \\
\text { (2006) } \\
\text { 3. Palmer D. (2006) } \\
\text { 4. Palmer D. (2007) } \\
\text { 5. Franks W., Gawn N., } \\
\text { \& Bowden G. (2007) } \\
\text { 6. Anstiss HD., \& } \\
\text { Ziaian T. (2010) } \\
\text { 7. Majumder P., } \\
\text { O'Reilly M., Karim K., } \\
\text { \& Vostanis P. (2014) } \\
\text { 8. Slewa-Younan et al. } \\
\text { (2014) }\end{array}$ \\
\hline & $\begin{array}{l}\text { Lack of trust of } \\
\text { authorities }\end{array}$ & $\begin{array}{l}\text { 1. Sri Lanka } \\
\text { 2. Somali } \\
\text { 3. Multiple } \\
\text { countries } \\
\text { 4. Multiple } \\
\text { countries } \\
\text { 5. Multiple } \\
\text { countries } \\
\text { 6. Multiple } \\
\text { countries } \\
\text { 7. Multiple } \\
\text { countries }\end{array}$ & $\begin{array}{l}\text { 1. Adults } \\
\text { 2. Adults } \\
\text { 3. Adults } \\
\text { 4. Adolescents } \\
\text { 5. Adults } \\
\text { 6. Youth } \\
\text { 7. Adults }\end{array}$ & $\begin{array}{l}\text { 1. Primary research } \\
\text { 2. Primary research } \\
\text { 3. Primary research } \\
\text { 4. Primary research } \\
\text { 5. Primary research } \\
\text { 6. Review } \\
\text { 7. Primary research }\end{array}$ & $\begin{array}{l}\text { 1. Beiser M., Simich } \\
\text { L., \& } \\
\text { Pandalangat N. (2003) } \\
\text { 2. Palmer D. (2006) } \\
\text { 3. Franks W., Gawn } \\
\text { N., \& } \\
\text { Bowden G. (2007) } \\
\text { 4. Anstiss HD., \& } \\
\text { Ziaian T. } \\
\text { (2010) } \\
\text { 5. Donnelly et al. } \\
\text { (2011) } \\
\text { 6. Ellis HB., Miller } \\
\text { AB., } \\
\text { Baldwin H., \& Abdi S. } \\
\text { (2011) } \\
\text { 7. Majumder P., } \\
\text { O’Reilly } \\
\text { M., Karim K., \& } \\
\text { Vostanis P. } \\
\text { (2014) }\end{array}$ \\
\hline
\end{tabular}

Table 2. to be Cont... 


\begin{tabular}{|c|c|c|c|c|}
\hline $\begin{array}{l}\text { Culturally oriented } \\
\text { health beliefs and } \\
\text { interpretation of the } \\
\text { causes of mental } \\
\text { illnesses }\end{array}$ & \begin{tabular}{|l} 
1. Multiple \\
countries \\
2. Somali \\
3. Multiple \\
countries \\
4. Multiple \\
countries \\
5. Multiple \\
countries \\
6. Sudan \\
\end{tabular} & $\begin{array}{l}\text { 1. Adults } \\
\text { 2. Adults } \\
\text { 3. Adolescents } \\
\text { 4. Adolescents } \\
\text { 5. Adolescents } \\
\text { 6. Adults }\end{array}$ & $\begin{array}{l}\text { 1. Commentary } \\
\text { 2. Primary research } \\
\text { 3. Primary research } \\
\text { 4. Primary research } \\
\text { 5. Primary research } \\
\text { 6. Primary research }\end{array}$ & $\begin{array}{l}\text { 1. Murphy et al. (2002) } \\
\text { 2. Palmer D. (2006) } \\
\text { 3. Anstiss et al. (2009) } \\
\text { 4. Anstiss HD., \& Ziaian T. (2010) } \\
\text { 5. Majumder P., O’Reilly } \\
\text { M., Karim K., \& Vostanis P. (2014) } \\
\text { 6. Savic M., Chur-Hansen A., } \\
\text { Mahmood MA., \& Moore V. (2016) }\end{array}$ \\
\hline $\begin{array}{l}\text { Lack of } \\
\text { understanding of } \\
\text { the process and } / \\
\text { or terminologies } \\
\text { of western mental } \\
\text { health treatmen }\end{array}$ & $\begin{array}{l}\text { 1. Somali } \\
\text { 2. Multiple } \\
\text { countries } \\
\text { 3. Multiple } \\
\text { countries } \\
\text { 4. Multiple } \\
\text { countries } \\
\text { 5. Multiple } \\
\text { countries }\end{array}$ & $\begin{array}{l}\text { 1. Adults } \\
\text { 2. Adults } \\
\text { 3. Adolescents } \\
\text { 4. Adults } \\
\text { 5. Adolescents }\end{array}$ & $\begin{array}{l}\text { 1. Primary research } \\
\text { 2. Primary research } \\
\text { 3. Primary research } \\
\text { 4. Primary research } \\
\text { 5. Primary research }\end{array}$ & $\begin{array}{l}\text { 1. Palmer D. (2006) } \\
\text { 2. Misra T., Connolly AM., \& } \\
\text { Majeed A. (2006) } \\
\text { 3. Anstiss et al. (2009) } \\
\text { 4. Donnelly et al. (2011) } \\
\text { 5. Majumder P., O'Reilly } \\
\text { M., Karim K., \& Vostanis P. (2014) }\end{array}$ \\
\hline $\begin{array}{l}\text { Lack of knowledge } \\
\text { of mental disorders } \\
\text { and the impact of } \\
\text { traumas on mental } \\
\text { wellbeing. }\end{array}$ & $\begin{array}{l}\text { 1. Multiple } \\
\text { countries } \\
\text { 2. Multiple } \\
\text { countries } \\
\text { 3. Multiple } \\
\text { countries } \\
\text { 4. Multiple } \\
\text { countries } \\
\text { 5. Iraq }\end{array}$ & $\begin{array}{l}\text { 1. Adults } \\
\text { 2. Adults } \\
\text { 3. Adolescents } \\
\text { 4. Adolescents } \\
\text { 5. Adults }\end{array}$ & $\begin{array}{l}\text { 1. Primary research } \\
\text { 2. Primary research } \\
\text { 3. Primary research } \\
\text { 4. Primary research } \\
\text { 5. Primary research }\end{array}$ & $\begin{array}{l}\text { 1. Misra T., Connolly AM., \& } \\
\text { Majeed A. (2006) } \\
\text { 2. Franks W., Gawn N., \& Bowden G. } \\
\text { (2007) } \\
\text { 3. Anstiss HD., \& Ziaian T. (2010) } \\
\text { 4. Majumder P., O’Reilly } \\
\text { M., Karim K., \& Vostanis P. (2014) } \\
\text { 5. Slewa-Younan et al. (2014) }\end{array}$ \\
\hline $\begin{array}{l}\text { Lack of recognition } \\
\text { of the importance of } \\
\text { mental healthcare* }\end{array}$ & $\begin{array}{l}\text { 1. Multiple } \\
\text { countries } \\
\text { 2. Multiple } \\
\text { countries } \\
\text { 3. Sudan }\end{array}$ & $\begin{array}{l}\text { 1. Adults } \\
\text { 2. Adolescents } \\
\text { 3. Adults }\end{array}$ & $\begin{array}{l}\text { 1. Primary research } \\
\text { 2. Primary research } \\
\text { 3. Primary research }\end{array}$ & $\begin{array}{l}\text { 1. Franks W., Gawn N., \& Bowden G. } \\
\text { (2007) } \\
\text { 2. Anstiss HD., \& Ziaian T. (2010) } \\
\text { 3. Savic M., Chur-Hansen A., Mahmood } \\
\text { MA., \& } \\
\text { Moore V. (2016) }\end{array}$ \\
\hline Language barriers & $\begin{array}{l}\text { 1. Multiple } \\
\text { countries } \\
\text { 2. Sri Lanka } \\
\text { 3. Multiple } \\
\text { countries } \\
\text { 4. Multiple } \\
\text { countries } \\
\text { 5. Multiple } \\
\text { countries }\end{array}$ & $\begin{array}{l}\text { 1. Adults } \\
\text { 2. Adults } \\
\text { 3. Adults } \\
\text { 4. Adults } \\
\text { 5. Youth }\end{array}$ & $\begin{array}{l}\text { 1. Primary research } \\
\text { 2. Primary research } \\
\text { 3. Primary research } \\
\text { 4. Primary research } \\
\text { 5. Review }\end{array}$ & $\begin{array}{l}\text { 1. Murphy et al. (2002) } \\
\text { 2. Beiser M., Simich L., \& } \\
\text { Pandalangat N. (2003) } \\
\text { 3. Misra T., Connolly AM., \& Majeed } \\
\text { A. (2006) } \\
\text { 4. Donnelly et al. (2011) } \\
\text { 5. Ellis HB., Miller AB., } \\
\text { Baldwin H., \& Abdi S. (2011) }\end{array}$ \\
\hline $\begin{array}{l}\text { Lack of social } \\
\text { support } \\
\text { utilization of } \\
\text { mental healthcare } \\
\text { services }\end{array}$ & $\begin{array}{l}\text { 1. Somali } \\
\text { 2. Multiple } \\
\text { countries } \\
\text { 3. Multiple } \\
\text { countries } \\
\end{array}$ & $\begin{array}{l}\text { 1. Adults } \\
\text { 2. Adolescents } \\
\text { 3. Youth }\end{array}$ & $\begin{array}{l}\text { 1. Primary research } \\
\text { 2. Systematic } \\
\text { review } \\
\text { 3. Review }\end{array}$ & $\begin{array}{l}\text { 1. Palmer D. (2006) } \\
\text { 2. Anstiss et al. (2009) } \\
\text { 3. Ellis HB., Miller AB., Baldwin H., \& } \\
\text { Abdi S. (2011) }\end{array}$ \\
\hline $\begin{array}{l}\text { Lack of awareness } \\
\text { of the availability } \\
\text { of mental healthcare } \\
\text { services }\end{array}$ & $\begin{array}{l}\text { 1. Multiple } \\
\text { countries } \\
\text { 2. Multiple } \\
\text { countries }\end{array}$ & $\begin{array}{l}\text { 1. Adults } \\
\text { 2. Adults }\end{array}$ & $\begin{array}{l}\text { 1. Commentary } \\
\text { 2. Primary research }\end{array}$ & $\begin{array}{l}\text { 1. Murphy et al. (2002) } \\
\text { 2. Donnelly et al. (2011) }\end{array}$ \\
\hline $\begin{array}{l}\text { Concerns about } \\
\text { confidentiality* }\end{array}$ & $\begin{array}{l}\text { 1. Somali } \\
\text { 2. Multiple } \\
\text { countries }\end{array}$ & $\begin{array}{l}\text { 1. Adults } \\
\text { 2. Adolescents }\end{array}$ & $\begin{array}{l}\text { 1. Primary research } \\
\text { 2. Primary research }\end{array}$ & $\begin{array}{l}\text { 1. Palmer D. (2007) } \\
\text { 2. Anstiss HD., \& Ziaian T. (2010) }\end{array}$ \\
\hline
\end{tabular}




\begin{tabular}{|c|c|c|c|c|c|}
\hline & $\begin{array}{l}\text { Concerns about } \\
\text { medical cost }\end{array}$ & $\begin{array}{l}\text { 1. Multiple } \\
\text { countries } \\
\text { 2. Multiple } \\
\text { countries }\end{array}$ & $\begin{array}{l}\text { 1. Adults } \\
\text { 2. Adolescents }\end{array}$ & $\begin{array}{l}\text { 1. Primary research } \\
\text { 2. Primary research }\end{array}$ & $\begin{array}{l}\text { 1. Franks W., Gawn N., \& } \\
\text { Bowden G. (2007) } \\
\text { 2. Anstiss et al. (2009) }\end{array}$ \\
\hline & Residential instability * & $\begin{array}{l}\text { 1. Somali } \\
\text { 2. Multiple } \\
\text { countries }\end{array}$ & $\begin{array}{l}\text { 1. Adults } \\
\text { 2. Adolescents }\end{array}$ & $\begin{array}{l}\text { 1. Primary research } \\
\text { 2. Primary research }\end{array}$ & $\begin{array}{l}\text { 1. Warfa et al. (2005) } \\
\text { 2. Anstiss et al. (2009) }\end{array}$ \\
\hline & $\begin{array}{l}\text { Fear of being in a } \\
\text { hospital* }\end{array}$ & $\begin{array}{l}\text { 1. Multiple } \\
\text { countries } \\
\text { 2. Multiple } \\
\text { countries }\end{array}$ & $\begin{array}{l}\text { 1. Adults } \\
\text { 2. Adolescents }\end{array}$ & $\begin{array}{l}\text { 1. Primary research } \\
\text { 2. Primary research }\end{array}$ & $\begin{array}{l}\text { 1. Franks W., Gawn N., \& } \\
\text { Bowden G. (2007) } \\
\text { 2. Majumder P., O’Reilly } \\
\text { M., Karim K., \& Vostanis } \\
\text { P. (2014) }\end{array}$ \\
\hline & $\begin{array}{l}\text { Inconvenience of } \\
\text { transportation }\end{array}$ & $\begin{array}{l}\text { 1. Sri Lanka } \\
\text { 2. Multiple } \\
\text { countries }\end{array}$ & $\begin{array}{l}\text { 1. Adults } \\
\text { 2. Adults }\end{array}$ & $\begin{array}{l}\text { 1. Primary research } \\
\text { 2. Primary research }\end{array}$ & $\begin{array}{l}\text { 1. Beiser M., Simich L., \& } \\
\text { Pandalangat N. (2003) } \\
\text { 2. Franks W., Gawn N., \& } \\
\text { Bowden G. (2007) }\end{array}$ \\
\hline & $\begin{array}{l}\text { Resistance to recall } \\
\text { traumatized memories* }\end{array}$ & $\begin{array}{l}\text { 1. Multiple } \\
\text { countries }\end{array}$ & 1. Adults & 1. Primary research & $\begin{array}{l}\text { 1. Franks W., Gawn N., \& } \\
\text { Bowden G. (2007) }\end{array}$ \\
\hline \multirow[t]{6}{*}{$\begin{array}{l}\text { Mental } \\
\text { Healthcare } \\
\text { System }\end{array}$} & $\begin{array}{l}\text { Lack of tailored } \\
\text { mental health services } \\
\text { for refugees }\end{array}$ & $\begin{array}{l}\text { 1. Sri Lanka } \\
\text { 2. Multiple } \\
\text { countries } \\
\text { 3. Multiple } \\
\text { countries } \\
\text { 4. Multiple } \\
\text { countries } \\
\text { 5. Multiple } \\
\text { countries } \\
\text { 6. Sudan }\end{array}$ & $\begin{array}{l}\text { 1. Adults } \\
\text { 2. Adults } \\
\text { 3. Adults } \\
\text { 4. Adults } \\
\text { 5. Youth } \\
\text { 6. Adults }\end{array}$ & $\begin{array}{l}\text { 1. Primary research } \\
\text { 2. Primary research } \\
\text { 3. Primary research } \\
\text { 4. Primary research } \\
\text { 5. Review } \\
\text { 6. Primary research }\end{array}$ & $\begin{array}{l}\text { 1. Beiser M., Simich L., \& Pandalangat } \\
\text { N. (2003) } \\
\text { 2. Ward K., \& Palmer D. (2005) } \\
\text { 3. Misra T., Connolly AM., \& Majeed } \\
\text { A. (2006) } \\
\text { 4. Donnelly et al. (2011) } \\
\text { 5. Ellis HB., Miller AB., Baldwin H., } \\
\text { \& Abdi S. (2011) } \\
\text { 6. Savic M., Chur-Hansen A., } \\
\text { Mahmood MA., \& Moore V. (2016) }\end{array}$ \\
\hline & $\begin{array}{l}\text { Lack of culturally } \\
\text { and/or linguistic } \\
\text { competent mental } \\
\text { healthcare providers }\end{array}$ & $\begin{array}{l}\text { 1. Multiple } \\
\text { countries } \\
\text { 2. Somali } \\
\text { 3. Multiple } \\
\text { countries } \\
\text { 4. Multiple } \\
\text { countries } \\
\text { 5. Multiple } \\
\text { countries } \\
\end{array}$ & $\begin{array}{l}\text { 1. Adults } \\
\text { 2. Adults } \\
\text { 3. Adolescents } \\
\text { 4. Adults } \\
\text { 5. Adolescents }\end{array}$ & $\begin{array}{l}\text { 1. Systematic } \\
\text { review } \\
\text { 2. Primary research } \\
\text { 3. Primary research } \\
\text { 4. Primary research } \\
\text { 5. Primary research }\end{array}$ & $\begin{array}{l}\text { 1. Feldman R. (2006) } \\
\text { 2. Palmer D. (2006) } \\
\text { 3. Anstiss et al. (2009) } \\
\text { 4. Donnelly et al. (2011) } \\
\text { 5. Majumder P., O'Reilly M., Karim } \\
\text { K., \& Vostanis P. (2014) }\end{array}$ \\
\hline & $\begin{array}{l}\text { Lack of mental } \\
\text { health } \\
\text { providers* }\end{array}$ & $\begin{array}{l}\text { 1. Sri Lanka } \\
\text { 2. Multiple } \\
\text { countries } \\
\text { 3. Multiple } \\
\text { countries } \\
\text { 4. Multiple } \\
\text { countries }\end{array}$ & $\begin{array}{l}\text { 1. Adults } \\
\text { 2. Adults } \\
\text { 3. Adults } \\
\text { 4. Adults }\end{array}$ & $\begin{array}{l}\text { 1. Primary research } \\
\text { 2. Primary research } \\
\text { 3. Systematic } \\
\text { review } \\
\text { 4. Primary research }\end{array}$ & $\begin{array}{l}\text { 1. Beiser M., Simich L., \& Pandalangat } \\
\text { N. (2003) } \\
\text { 2. Ward K., \& Palmer D. (2005) } \\
\text { 3. Feldman R. (2006) } \\
\text { 4. Franks W., Gawn N., } \\
\text { \& Bowden G. (2007) }\end{array}$ \\
\hline & $\begin{array}{l}\text { Lack of professional } \\
\text { interpreters } \quad \text { and/or } \\
\text { translator }\end{array}$ & $\begin{array}{l}\text { 1. Multiple } \\
\text { countries } \\
\text { 2. Multiple } \\
\text { countries } \\
\text { 3. Multiple } \\
\text { countries }\end{array}$ & $\begin{array}{l}\text { 1. Adults } \\
\text { 2. Adults } \\
\text { 3. Adults }\end{array}$ & $\begin{array}{l}\text { 1. Commentary } \\
\text { 2. Primary research } \\
\text { 3. Primary research }\end{array}$ & $\begin{array}{l}\text { 1. Murphy et al. (2002) } \\
\text { 2. Franks W., Gawn N., \& Bowden G. } \\
\text { (2007) } \\
\text { 3. Donnelly et al. (2011) }\end{array}$ \\
\hline & $\begin{array}{l}\text { Discriminative } \\
\text { attitudes and beliefs } \\
\text { in practice* }\end{array}$ & $\begin{array}{l}\text { 1. Somali } \\
\text { 2. Multiple } \\
\text { countries }\end{array}$ & $\begin{array}{l}\text { 1. Adults } \\
\text { 2. Adolescents }\end{array}$ & $\begin{array}{l}\text { 1. Primary research } \\
\text { 2. Systematic } \\
\text { review }\end{array}$ & $\begin{array}{l}\text { 1. Palmer D. (2006) } \\
\text { 2. Anstiss et al. (2009) }\end{array}$ \\
\hline & $\begin{array}{l}\text { Lack of consideration of } \\
\text { social, political and } \\
\text { economic factors in } \\
\text { mental healthcare } \\
\text { services* }\end{array}$ & $\begin{array}{l}\text { 1. Multiple } \\
\text { countries }\end{array}$ & 1.Not specified & 1. Review & 1. Watters C. (2001) \\
\hline
\end{tabular}


In particular, in other western countries, the literature suggested how parental decision could impact their children and/or adolescents' use of mental healthcare and emphasized the needs of involving parents in psychoeducation [41,47,51]. It described how a hospital environment could intimidate refugee patients and make them feel like a "cage" [43,45]. It also described the refugee patients' reluctance of recalling their traumatized memories [43]. Furthermore, few refugee groups identified that residential instability contributed to their underutilization of mental healthcare [47,52], as well as identified the relationship between low family priority placed on mental health and the receipt of services $[43,44,48]$.

In responding to the challenges faced by the refugees in western countries to utilize the mental healthcare, four themes emerged from the current literature that implied the potential solutions to the effectiveness of addressing mental disorders among refugee populations (Table 3): (1) Adopt effective westernized mental health treatment Psychotherapy has proven its effectiveness among refugee populations, which included Cognitive Behavioral Therapy
(CBT), Narrative Exposure Therapy (NET), Cognitive Processing Therapy, Imagery Rescripting Therapy (ImRs), Treatment As Usual, Eye Movement Desensitization and Processing (EMDR), and other trauma-focused therapies/interventions [56-66]. A systematic review of 25 studies demonstrated the suitability of standard CBT and therapies with CBT forms for treating PTSD among adult refugee populations [58]. Ten randomized clinical trials including both refugee and asylum-seeker PTSD patients with multiple traumatic experiences were also critically reviewed. Both NET and CBT presented moderate evidence of support to the effectiveness of the treatment among both populations [57]. Most recent literature indicated the effectiveness of both ImRs and EMDR among refugees. Ten refugee PTSD patients in Netherland were invited for ImRs, which started with a memory of a traumatic or stressful event and then imagined an intervention in order to achieve a better outcome. Except for one patient, there was a significant improvement of PTSD symptoms among the rest of the group [64]. EMDR also started with a refugee participant recalling a traumatic experience but simultaneously

\begin{tabular}{|c|c|c|c|c|c|}
\hline Relevance & $\begin{array}{l}\text { Approaches to } \\
\text { Mental Healthcare }\end{array}$ & $\begin{array}{l}\text { Target Refugee } \\
\text { Population }\end{array}$ & $\begin{array}{l}\text { Study Population } \\
\text { Age }\end{array}$ & Research Design & $\begin{array}{l}\text { Authors and Year } \\
\text { of Publication }\end{array}$ \\
\hline \multirow[t]{8}{*}{$\begin{array}{l}\text { Western Mental } \\
\text { Healthcare }\end{array}$} & $\begin{array}{l}\text { Cognitive } \\
\text { Behavioral } \\
\text { Therapy }\end{array}$ & $\begin{array}{l}\text { 1. Multiple } \\
\text { countries } \\
\text { 2. Multiple } \\
\text { countries } \\
\text { 3. Multiple } \\
\text { countries } \\
\text { 4. Multiple } \\
\text { countries }\end{array}$ & $\begin{array}{l}\text { 1. Not specified } \\
\text { 2. Not specified } \\
\text { 3. Adults } \\
\text { 4. Not specified }\end{array}$ & $\begin{array}{l}\text { 1. Review } \\
\text { 2. Systematic } \\
\text { review } \\
\text { 3. Systematic } \\
\text { review } \\
\text { 4. Systematic } \\
\text { review }\end{array}$ & $\begin{array}{l}\text { 1. Murray KE., } \\
\text { Davidson GR., \& } \\
\text { Schweitzer RD. } \\
\text { (2010) } \\
\text { 2. Crumlish N., \& } \\
\text { Rourke KO. (2010) } \\
\text { 3. Palic S., \& Elklit } \\
\text { A. (2010) } \\
\text { 4. Slobodin O., \& } \\
\text { de Jong J. (2014) }\end{array}$ \\
\hline & $\begin{array}{l}\text { Narrative Exposure } \\
\text { Therapy }\end{array}$ & $\begin{array}{l}\text { 1. Multiple } \\
\text { countries } \\
\text { 2. Multiple } \\
\text { countries } \\
\text { 3. Multiple } \\
\text { countries }\end{array}$ & $\begin{array}{l}\text { 1. Not specified } \\
\text { 2. Adults } \\
\text { 3. Not specified }\end{array}$ & $\begin{array}{l}\text { 1. Systematic } \\
\text { review } \\
\text { 2. Primary research } \\
\text { 3. Systematic } \\
\text { review }\end{array}$ & $\begin{array}{l}\text { 1. Crumlish N., \& } \\
\text { Rourke KO. (2010) } \\
\text { 2. Stenmark et al. } \\
\text { (2013) } \\
\text { 3. Slobodin O., \& } \\
\text { de Jong J. (2014) }\end{array}$ \\
\hline & $\begin{array}{l}\text { Cognitive } \\
\text { Processing Therapy }\end{array}$ & $\begin{array}{l}\text { 1. Bosnia } \\
\text { 2. Multiple } \\
\text { countries }\end{array}$ & $\begin{array}{l}\text { 1. Adults } \\
\text { 2. Adults }\end{array}$ & $\begin{array}{l}\text { 1. Case report } \\
\text { 2. Primary research }\end{array}$ & $\begin{array}{l}\text { 1. Schulz PM., } \\
\text { Huber CL., \& } \\
\text { Resick PA. (2006) } \\
\text { 2. Schulz PM., } \\
\text { Resick PA., Huber } \\
\text { CL., \& Griffin MG. } \\
\text { (2006) }\end{array}$ \\
\hline & $\begin{array}{l}\text { Trauma-focused } \\
\text { therapies/ } \\
\text { interventions }\end{array}$ & $\begin{array}{l}\text { 1. Multiple } \\
\text { countries }\end{array}$ & 1. Not specified & $\begin{array}{l}\text { 1. Systematic } \\
\text { review }\end{array}$ & $\begin{array}{l}\text { 1. Nickerson A., } \\
\text { Bryant RA., Silove } \\
\text { D., \& Steel Z. } \\
(2010)\end{array}$ \\
\hline & $\begin{array}{l}\text { Imagery } \\
\text { Rescripting } \\
\text { Therapy }\end{array}$ & $\begin{array}{l}\text { 1. Multiple } \\
\text { countries }\end{array}$ & 1. Adults & 1. Primary research & $\begin{array}{l}\text { 1. Arntz A., Sofi } \\
\text { D., \& Breukelen } \\
\text { GV. (2013) }\end{array}$ \\
\hline & $\begin{array}{l}\text { Treatment As } \\
\text { Usual }\end{array}$ & $\begin{array}{l}\text { 1. Multiple } \\
\text { countries }\end{array}$ & 1. Adults & 1. Primary research & $\begin{array}{l}\text { 1. Arntz A., Sofi } \\
\text { D., \& Breukelen } \\
\text { GV. (2013) }\end{array}$ \\
\hline & $\begin{array}{l}\text { Eye Movement } \\
\text { Desensitization and } \\
\text { Processing }\end{array}$ & 1. Syria & 1. Adults & 1. Primary research & $\begin{array}{l}\text { 1. Acarturk et al. } \\
\text { (2015) }\end{array}$ \\
\hline & Psychotherapy & 1. Bosnia & 1. Adults & 1. Primary research & $\begin{array}{l}\text { 1. Weine et al. } \\
\text { (2001) }\end{array}$ \\
\hline
\end{tabular}




\begin{tabular}{|c|c|c|c|c|c|}
\hline \multirow[t]{2}{*}{$\begin{array}{l}\text { Culturally } \\
\text { Relevant } \\
\text { Mental Health } \\
\text { Coping }\end{array}$} & Religion & $\begin{array}{l}\text { 1. Somali } \\
\text { 2. Somali } \\
\text { 3. Somali } \\
\text { 4. Sudan } \\
\text { 5. Tibet } \\
\text { 6. Multiple } \\
\text { countries } \\
\text { 7. Somali } \\
\text { 8. Iraq } \\
\text { 9. Somali }\end{array}$ & $\begin{array}{l}\text { 1. Adults } \\
\text { 2. Adults and } \\
\text { adolescents } \\
\text { 3. Adults } \\
\text { 4. Adults } \\
\text { 5. Adults and } \\
\text { adolescents } \\
\text { 6. Not specified } \\
\text { 7. Adults } \\
\text { 8. Adults } \\
\text { 9. Adults }\end{array}$ & $\begin{array}{l}\text { 1. Primary research } \\
\text { 2. Primary research } \\
\text { 3. Primary research } \\
\text { 4. Primary research } \\
\text { 5. Primary research } \\
\text { 6. Systematic review } \\
\text { 7. Primary research } \\
\text { 8. Primary research } \\
\text { 9. Primary research }\end{array}$ & $\begin{array}{l}\text { 1. Carroll JK. (2004) } \\
\text { 2. Whittaker S., Hardy G., Lewis K., \& } \\
\text { Buchan L. (2005) } \\
\text { 3. Palmer D. (2007) } \\
\text { 4. Khawaja NG., White KM., } \\
\text { Schweitzer R., \& Greenslade JH. } \\
\text { (2008) } \\
\text { 5. Sachs et al. (2008) } \\
\text { 6. MacDuff S., Grodin MA., \& } \\
\text { Gardiner P. (2011) } \\
\text { 7. Longacre M., Silver-Highfield E., } \\
\text { Lama P., \& Grodin MA. (2012) } \\
\text { 8. Slewa-Younan et al. (2014) } \\
\text { 9. Bettmann JE., Penney D., Freeman } \\
\text { PC., \& Lecy N. (2015) }\end{array}$ \\
\hline & Social support & $\begin{array}{l}\text { 1. Multiple } \\
\text { countries } \\
\text { 2. Somali } \\
\text { 3. Somali } \\
\text { 4. Somali } \\
\text { 5. Sudan } \\
\text { 6. Tibet } \\
\text { 7. Multiple } \\
\text { countries } \\
\text { 8. Somali } \\
\text { 9. Multiple } \\
\text { countries }\end{array}$ & $\begin{array}{l}\text { 1. Adults } \\
\text { 2. Adults } \\
\text { 3. Adults } \\
\text { 4. Adults and } \\
\text { adolescents } \\
\text { 5. Adults } \\
\text { 6. Adults } \\
\text { 7. Adolescents } \\
\text { 8. Adults } \\
\text { 9. Adults }\end{array}$ & $\begin{array}{l}\text { 1. Primary research } \\
\text { 2. Primary research } \\
\text { 3. Primary research } \\
\text { 4. Primary research } \\
\text { 5. Primary research } \\
\text { 6. Primary research } \\
\text { 7. Primary research } \\
\text { 8. Primary research } \\
\text { 9. Primary research }\end{array}$ & $\begin{array}{l}\text { 1. Behnia B. (2003) } \\
\text { 2. Carroll JK. (2004) } \\
\text { 3. Guerin B., Guerin P., Diiriye RO., \& } \\
\text { Yates S. (2004) } \\
\text { 4. Whittaker S., Hardy G., Lewis K., \& } \\
\text { Buchan L. (2005) } \\
\text { 5. Khawaja NG., White KM., } \\
\text { Schweitzer R., \& Greenslade JH. } \\
\text { (2008) } \\
\text { 6. Sachs et al. (2008) } \\
\text { 7. Anstiss HD., \& Ziaian T. (2010) } \\
\text { 8. Longacre M., Silver-Highfield E., } \\
\text { Lama P., \& Grodin MA. (2012) } \\
\text { 9. Mitschke et al. (2018) }\end{array}$ \\
\hline & $\begin{array}{l}\text { Relaxation } \\
\text { techniques } \\
\text { (e.g., massage, } \\
\text { meditation) }\end{array}$ & $\begin{array}{l}\text { 1. Multiple } \\
\text { countries } \\
\text { 2. Somali } \\
\text { 3. Somali } \\
\text { 4. Multiple } \\
\text { countries } \\
\text { 5. Multiple } \\
\text { countries }\end{array}$ & $\begin{array}{l}\text { 1. Adults } \\
\text { 2. Adults } \\
\text { 3. Adults } \\
\text { 4. Not specified } \\
\text { 5. Not specified }\end{array}$ & $\begin{array}{l}\text { 1. Primary research } \\
\text { 2. Primary research } \\
\text { 3. Primary research } \\
\text { 4. Systematic review } \\
\text { 5. Systematic review }\end{array}$ & $\begin{array}{l}\text { 1.Behnia B. (2003) } \\
\text { 2. Guerin B., Guerin P., Diiriye RO., \& } \\
\text { Yates S. (2004) } \\
\text { 3. Palmer D. (2007) } \\
\text { 4. MacDuff S., Grodin MA., \& } \\
\text { Gardiner P. (2011) } \\
\text { 5. Longacre M., Silver-Highfield E., } \\
\text { Lama P., \& Grodin MA. (2012) }\end{array}$ \\
\hline & $\begin{array}{l}\text { Traditional } \\
\text { medication }\end{array}$ & $\begin{array}{l}\text { 1. Southeast } \\
\text { Asia } \\
\text { 2. Somali } \\
\text { 3. Somali } \\
\text { 4. Multiple } \\
\text { countries }\end{array}$ & $\begin{array}{l}\text { 1. Adults } \\
\text { 2. Adults } \\
\text { 3. Adults } \\
\text { 4. Not specified }\end{array}$ & $\begin{array}{l}\text { 1. Primary research } \\
\text { 2. Primary research } \\
\text { 3. Primary research } \\
\text { 4. Systematic review }\end{array}$ & $\begin{array}{l}\text { 1. Chung RC., \& Lin K. (1994) } \\
\text { 2. Carroll JK. (2004) } \\
\text { 3. Palmer D. (2007) } \\
\text { 4. Longacre M., Silver-Highfield E., } \\
\text { Lama P., \& Grodin MA. (2012) }\end{array}$ \\
\hline & $\begin{array}{l}\text { Music therapy } \\
\text { or dance } \\
\text { movement }\end{array}$ & $\begin{array}{l}\text { 1. Multiple } \\
\text { countries } \\
\text { 2. Multiple } \\
\text { countries } \\
\text { 3. Multiple } \\
\text { countries } \\
\text { 4. Somali }\end{array}$ & $\begin{array}{l}\text { 1. Adults } \\
\text { 2. Not specified } \\
\text { 3. Not specified } \\
\text { 4. Adults }\end{array}$ & $\begin{array}{l}\text { 1. Primary research } \\
\text { 2. Systematic review } \\
\text { 3. Systematic review } \\
\text { 4. Primary research }\end{array}$ & $\begin{array}{l}\text { 1.Behnia B. (2003) } \\
\text { 2. MacDuff S., Grodin MA., \& } \\
\text { Gardiner P. (2011) } \\
\text { 3. Longacre M., Silver-Highfield E., } \\
\text { Lama P., \& Grodin MA. (2012) } \\
\text { 4. Palmer D. (2007) }\end{array}$ \\
\hline & Acupuncture & $\begin{array}{l}\text { 1. Multiple } \\
\text { countries } \\
\text { 2. Multiple } \\
\text { countries } \\
\text { 3. Multiple } \\
\text { countries }\end{array}$ & $\begin{array}{l}\text { 1. Adults } \\
\text { 2. Not specified } \\
\text { 3. Not specified }\end{array}$ & $\begin{array}{l}\text { 1. Case report } \\
\text { 2. Systematic review } \\
\text { 3. Systematic review }\end{array}$ & $\begin{array}{l}\text { 1. Pease M., Sollom R., \& Wayne P. } \\
\text { (2009) } \\
\text { 2. MacDuff S., Grodin MA., \& } \\
\text { Gardiner P. (2011) } \\
\text { 3. Longacre M., Silver-Highfield E., } \\
\text { Lama P., \& Grodin MA. (2012) }\end{array}$ \\
\hline & $\begin{array}{l}\text { Improving } \\
\text { diet and/or } \\
\text { exercise }\end{array}$ & $\begin{array}{l}\text { 1. Iraq } \\
\text { 2. Multiple } \\
\text { countries } \\
\text { 3. Somali }\end{array}$ & $\begin{array}{l}\text { 1. Adults } \\
\text { 2. Adults } \\
\text { 3. Adults }\end{array}$ & $\begin{array}{l}\text { 1. Primary research } \\
\text { 2. Primary research } \\
\text { 3. Primary research }\end{array}$ & $\begin{array}{l}\text { 1. Slewa-Younan et al. (2014) } \\
\text { 2. Behnia B. (2003) } \\
\text { 3. Palmer D. (2007) }\end{array}$ \\
\hline
\end{tabular}

Table 3. to be Cont.... 


\begin{tabular}{|c|c|c|c|c|c|}
\hline & $\begin{array}{l}\text { Positive attitude and } \\
\text { belief in inner strength }\end{array}$ & $\begin{array}{l}\text { 1. Somali } \\
\text { 2. Sudan }\end{array}$ & $\begin{array}{l}\text { 1. Adults and } \\
\text { adolescents } \\
\text { 2. Adults }\end{array}$ & $\begin{array}{l}\text { 1. Primary research } \\
\text { 2. Primary research }\end{array}$ & $\begin{array}{l}\text { 1. Whittaker S., Hardy G., } \\
\text { Lewis K., \& Buchan L. } \\
\text { (2005) } \\
\text { 2. Khawaja NG., White } \\
\text { KM., Schweitzer R., \& } \\
\text { Greenslade JH. (2008) }\end{array}$ \\
\hline & Tai-chi and Qigong & $\begin{array}{l}\text { 1. Multiple } \\
\text { countries } \\
\text { 2. Multiple } \\
\text { countries }\end{array}$ & $\begin{array}{l}\text { 1. Not specified } \\
\text { 2. Not specified }\end{array}$ & $\begin{array}{l}\text { 1. Systematic review } \\
\text { 2. Systematic review }\end{array}$ & $\begin{array}{l}\text { Gardiner P. (2011) } \\
\text { 2. Longacre M., Silver- } \\
\text { Highfield E., Lama P., \& } \\
\text { Grodin MA. (2012) }\end{array}$ \\
\hline & Traditional healer & $\begin{array}{l}\text { 1. Haiti } \\
\text { 2. Multiple } \\
\text { countries }\end{array}$ & $\begin{array}{l}\text { 1. Not specified } \\
\text { 2. Not specified }\end{array}$ & $\begin{array}{l}\text { 1. Commentary } \\
\text { 2. Systematic review }\end{array}$ & $\begin{array}{l}\text { 1. Gustafson MB. (1989) } \\
\text { 2. MacDuff S., Grodin MA., } \\
\text { \& Gardiner P. (2011) }\end{array}$ \\
\hline & Home remedies & 1. Haiti & 1. Not specified & 1. Commentary & 1. Gustafson MB. (1989) \\
\hline & $\begin{array}{l}\text { Reading a self-help } \\
\text { book }\end{array}$ & 1. Iraq & 1. Adults & 1. Primary research & $\begin{array}{l}\text { 1. Slewa-Younan et al. } \\
\text { (2014) }\end{array}$ \\
\hline & Finding new hobbies & 1. Iraq & 1. Adults & 1. Primary research & $\begin{array}{l}\text { 1. Slewa-Younan et al. } \\
(2014)\end{array}$ \\
\hline & Crying & $\begin{array}{l}\text { 1. Multiple } \\
\text { countries }\end{array}$ & 1. Adults & 1. Primary research & 1.Behnia B. (2003) \\
\hline & Self-analysis & $\begin{array}{l}\text { 1. Multiple } \\
\text { countries }\end{array}$ & 1. Adults & 1. Primary research & 1.Behnia B. (2003) \\
\hline & $\begin{array}{l}\text { Reading and/or writing } \\
\text { diaries or poems }\end{array}$ & $\begin{array}{l}\text { 1. Multiple } \\
\text { countries }\end{array}$ & 1. Adults & 1. Primary research & 1.Behnia B. (2003) \\
\hline & Drinking & $\begin{array}{l}\text { 1. Multiple } \\
\text { countries }\end{array}$ & 1. Adults & 1. Primary research & 1.Behnia B. (2003) \\
\hline & Embodied metaphors & 1. Sudan & 1. Adults & 1. Primary research & 1. Coker EM (2004) \\
\hline & $\begin{array}{l}\text { Acceptance of the past } \\
\text { and focusing on the } \\
\text { future }\end{array}$ & 1. Sudan & 1. Adults & 1. Primary research & $\begin{array}{l}\text { 1. Khawaja NG., White } \\
\text { KM., Schweitzer R., \& } \\
\text { Greenslade JH. (2008) }\end{array}$ \\
\hline \multirow[t]{2}{*}{$\begin{array}{l}\text { Address and } \\
\text { Remove } \\
\text { Existing } \\
\text { Barriers } \\
\text { to Mental } \\
\text { Healthcare }\end{array}$} & $\begin{array}{l}\text { Family and community } \\
\text { interventions on } \\
\text { education of mental } \\
\text { health to gain family } \\
\text { and community support } \\
\text { of mental healthcare }\end{array}$ & $\begin{array}{l}\text { 1. Southeast } \\
\text { Asia } \\
\text { 2. Asia } \\
\text { 3. Somali } \\
\text { 4. Somali } \\
\text { 5. Bosnia } \\
\text { 6. Multiple } \\
\text { countries } \\
\text { 7. Multiple } \\
\text { countries }\end{array}$ & $\begin{array}{l}\text { 1. Not specified } \\
\text { 2. Not specified } \\
\text { 3. Adults } \\
\text { 4. Adults } \\
\text { 5. Adults } \\
\text { 6. Youth } \\
\text { 7. Not specified }\end{array}$ & $\begin{array}{l}\text { 1. Case report } \\
\text { 2. Review } \\
\text { 3. Primary research } \\
\text { 4. Primary research } \\
\text { 5. Primary research } \\
\text { 6. Review } \\
\text { 7. Case report }\end{array}$ & $\begin{array}{l}\text { 1. True RH. (1985) } \\
\text { 2. Dhooper SS., \& Tran TV. } \\
\text { (1998) } \\
\text { 3. Guerin B., Guerin P., } \\
\text { Diiriye RO., \& Yates S. } \\
\text { (2004) } \\
\text { 4. Palmer D. (2007) } \\
\text { 5. Weine et al. (2008) } \\
\text { 6. Ellis HB., Miller AH., } \\
\text { \& Baldwin H., \& Abdi S. } \\
\text { (2011) } \\
\text { 7. Nazzal et al. (2014) }\end{array}$ \\
\hline & $\begin{array}{l}\text { Education for refugee } \\
\text { patients about mental } \\
\text { health and western } \\
\text { treatment and mental } \\
\text { healthcare system }\end{array}$ & $\begin{array}{l}\text { 1. Southeast } \\
\text { Asia } \\
\text { 2. Asia } \\
\text { 3. Cambodia } \\
\text { 4. Multiple } \\
\text { countries } \\
\text { 5. Multiple } \\
\text { countries } \\
\text { 6. Multiple } \\
\text { countries }\end{array}$ & $\begin{array}{l}\text { 1. Adults } \\
\text { 2. Not specified } \\
\text { 3. Not specified } \\
\text { 4. Adult } \\
\text { 5. Not specified } \\
\text { 6. Adults }\end{array}$ & $\begin{array}{l}\text { 1. Case report } \\
\text { 2. Review } \\
\text { 3. Review } \\
\text { 4. Case report } \\
\text { 5. Case report } \\
\text { 6. Primary research }\end{array}$ & $\begin{array}{l}\text { 1. Allden K. (1998) } \\
\text { 2. Dhooper SS., \& Tran TV. } \\
\text { (1998) } \\
\text { 3. Chung RC. (2001) } \\
\text { 4. d'Ardenne P., Capuzzo } \\
\text { N., Ruaro L., \& Priebe S. } \\
\text { (2005) } \\
\text { 5. Nazzal et al. (2014) } \\
\text { 6. Shannon PJ., Vinson GA., } \\
\text { Cook TL., \& Lennon E. } \\
\text { (2016) }\end{array}$ \\
\hline
\end{tabular}

Table 3. to be Cont.... 


\begin{tabular}{|c|c|c|c|c|}
\hline $\begin{array}{l}\text { Cross-cultural } \\
\text { psychiatric/competency } \\
\text { training for mental } \\
\text { healthcare practitioner } \\
\text { and staff }\end{array}$ & $\begin{array}{l}\text { 1. Southeast } \\
\text { Asia } \\
\text { 2. Asia } \\
\text { 3. Multiple } \\
\text { countries } \\
\text { 4. Multiple } \\
\text { countries } \\
\text { 5. Multiple } \\
\text { countries }\end{array}$ & $\begin{array}{l}\text { 1. Not specified } \\
\text { 2. Not specified } \\
\text { 3. Not specified } \\
\text { 4. Not specified } \\
\text { 5. Adults }\end{array}$ & $\begin{array}{l}\text { 1. Case report } \\
\text { 2. Review } \\
\text { 3. Commentary } \\
\text { 4. Review } \\
\text { 5. Primary research }\end{array}$ & $\begin{array}{l}\text { 1. True RH. (1985) } \\
\text { 2. Dhooper SS., \& Tran TV. } \\
\text { (1998) } \\
\text { 3. Burnett A., \& Gebremikael L. } \\
\text { (2005) } \\
\text { 4. Blight KJ., Ekblad S., } \\
\text { Lindencrona F., \& Shahnavaz S. } \\
\text { (2009) } \\
\text { 5. Bäärnhielm S., Edlund A., } \\
\text { Loannou M., \& Dahlin M. (2014) }\end{array}$ \\
\hline $\begin{array}{l}\text { Partnership of } \\
\text { healthcare practitioners } \\
\text { and cultural experts }\end{array}$ & $\begin{array}{l}\text { 1. Multiple } \\
\text { countries } \\
\text { 2. Somali } \\
\text { 3. Multiple } \\
\text { countries } \\
\text { 4. Multiple } \\
\text { countries }\end{array}$ & $\begin{array}{l}\text { 1. Adults } \\
\text { 2. Adults } \\
\text { 3. Youth } \\
\text { 4. Adults }\end{array}$ & $\begin{array}{l}\text { 1. Case report } \\
\text { 2. Primary research } \\
\text { 3. Review } \\
\text { 4. Primary research }\end{array}$ & $\begin{array}{l}\text { 1. Kirmayer et al. (2003) } \\
\text { 2. Guerin B., Guerin P., Diiriye } \\
\text { RO., \& Yates S. (2004) } \\
\text { 3. Ellis HB., Miller AH., \& } \\
\text { Baldwin H., \& Abdi S. (2011) } \\
\text { 4. Shannon PJ. (2014) }\end{array}$ \\
\hline $\begin{array}{l}\text { Building a trustworthy } \\
\text { relationship between } \\
\text { patients and } \\
\text { practitioners }\end{array}$ & $\begin{array}{l}\text { 1. Southeast } \\
\text { Asia } \\
\text { 2. Multiple } \\
\text { countries } \\
\text { 3. Multiple } \\
\text { countries } \\
\text { 4. Multiple } \\
\text { countries }\end{array}$ & $\begin{array}{l}\text { 1. Not specified } \\
\text { 2. Not specified } \\
\text { 3. Adults } \\
\text { 4. Adults }\end{array}$ & $\begin{array}{l}\text { 1. Case report } \\
\text { 2. Review } \\
\text { 3. Primary research } \\
\text { 4. Primary research }\end{array}$ & $\begin{array}{l}\text { 1. Murase K., Egawa J., \& } \\
\text { Tashima N. (1985) } \\
\text { 2. Crosby SS. (2013) } \\
\text { 3. Shannon PJ., Vinson GA., } \\
\text { Cook TL., \& Lennon E. (2016) } \\
\text { 4. Mitschke et al. (2018) }\end{array}$ \\
\hline $\begin{array}{l}\text { Increased use } \\
\text { of professional } \\
\text { interpreters/translators }\end{array}$ & $\begin{array}{l}\text { 1. Somali } \\
\text { 2. Multiple } \\
\text { countries } \\
\text { 3. Multiple } \\
\text { countries } \\
\text { 4. Multiple } \\
\text { countries }\end{array}$ & $\begin{array}{l}\text { 1. Adults } \\
\text { 2. Not specified } \\
\text { 3. Adults } \\
\text { 4. Not specified }\end{array}$ & $\begin{array}{l}\text { 1. Primary research } \\
\text { 2. Commentary } \\
\text { 3. Case report } \\
\text { 4. Review }\end{array}$ & $\begin{array}{l}\text { 1. Guerin BWV., Guerin P., } \\
\text { Diiriye RO., \& Yates S. (2004) } \\
\text { 2. Burnett A., \& Gebremikael L. } \\
\text { (2005) } \\
\text { 3. d'Ardenne P., Capuzzo N., } \\
\text { Ruaro L., \& Priebe S. (2005) } \\
\text { 4. Crosby SS. (2013) }\end{array}$ \\
\hline $\begin{array}{l}\text { Integration of mental } \\
\text { health services in } \\
\text { existing service } \\
\text { systems to reduce } \\
\text { stigma (e.g., school, } \\
\text { primary care) }\end{array}$ & $\begin{array}{l}\text { 1. Multiple } \\
\text { countries } \\
\text { 2. Multiple } \\
\text { countries } \\
\text { 3. Somali }\end{array}$ & $\begin{array}{l}\text { 1. Children and } \\
\text { adolescents } \\
\text { 2. Youth } \\
\text { 3. Adults }\end{array}$ & $\begin{array}{l}\text { 1. Primary research } \\
\text { 2. Review } \\
\text { 3. Primary research }\end{array}$ & $\begin{array}{l}\text { 1. Fazel M., Doll H., \& Stein A. } \\
\text { (2009) } \\
\text { 2. Ellis HB., Miller AH., \& } \\
\text { Baldwin H., \& Abdi S. (2011) } \\
\text { 3. White CC., Solid CA., Hodges } \\
\text { JS., \& Boehm DH. (2015) }\end{array}$ \\
\hline $\begin{array}{l}\text { Enhancement of } \\
\text { culturally responsive } \\
\text { services }\end{array}$ & $\begin{array}{l}\text { 1. Southeast } \\
\text { Asia } \\
\text { 2. Cambodia } \\
\text { 3. Multiple } \\
\text { countries }\end{array}$ & $\begin{array}{l}\text { 1. Adults } \\
\text { 2. Not specified } \\
\text { 3. Adults }\end{array}$ & $\begin{array}{l}\text { 1. Case report } \\
\text { 2. Review } \\
\text { 3. Primary research }\end{array}$ & $\begin{array}{l}\text { 1. Allden K. (1998) } \\
\text { 2. Chung RC. (2001) } \\
\text { 3. Shannon PJ., Vinson GA., } \\
\text { Cook TL., \& Lennon E. (2016) }\end{array}$ \\
\hline $\begin{array}{l}\text { Integration of } \\
\text { ethnography approach } \\
\text { in multicultural mental } \\
\text { healthcare practice }\end{array}$ & $\begin{array}{l}\text { 1. Multiple } \\
\text { countries } \\
\text { 2. Bhutan }\end{array}$ & $\begin{array}{l}\text { 1. Not specified } \\
\text { 2. Not specified }\end{array}$ & $\begin{array}{l}\text { 1. Review } \\
\text { 2. Review }\end{array}$ & $\begin{array}{l}\text { 1. Goździak EM. (2004) } \\
\text { 2. Kohrt BA., Maharjan SM., } \\
\text { Timsina D., \& Griffith JL. (2012) }\end{array}$ \\
\hline $\begin{array}{l}\text { Integration of western } \\
\text { and traditional } \\
\text { treatment }\end{array}$ & $\begin{array}{l}\text { 1. Cambodia } \\
\text { 2. Multiple } \\
\text { countries }\end{array}$ & $\begin{array}{l}\text { 1. Not specified } \\
\text { 2. Not specified }\end{array}$ & $\begin{array}{l}\text { 1. Review } \\
\text { 2. Review }\end{array}$ & $\begin{array}{l}\text { 1. Chung RC. (2001) } \\
\text { 2. Crosby SS. (2013) }\end{array}$ \\
\hline $\begin{array}{l}\text { Easy access to mental } \\
\text { healthcare facilities }\end{array}$ & $\begin{array}{l}\text { 1. Multiple } \\
\text { countries } \\
\text { 2. Multiple } \\
\text { countries }\end{array}$ & $\begin{array}{l}\text { 1. Adults } \\
\text { 2. Adults }\end{array}$ & $\begin{array}{l}\text { 1. Primary research } \\
\text { 2. Primary research }\end{array}$ & $\begin{array}{l}\text { 1. Shannon PJ., Vinson GA., } \\
\text { Cook TL., \& Lennon E. (2016) } \\
\text { 2. Mitschke et al. (2018) }\end{array}$ \\
\hline $\begin{array}{l}\text { Empowerment of } \\
\text { refugees with new } \\
\text { culture }\end{array}$ & 1. Cambodia & 1. Not specified & 1. Review & 1. Chung RC. (2001) \\
\hline
\end{tabular}

Table 3. to be Cont.... 


\begin{tabular}{|c|c|c|c|c|c|}
\hline & $\begin{array}{l}\text { Improvement of refugees' } \\
\text { foreign language } \\
\text { proficiency }\end{array}$ & $\begin{array}{l}\text { 1. Multiple } \\
\text { countries }\end{array}$ & 1. Adults & $\begin{array}{l}\text { 1. Primary } \\
\text { research }\end{array}$ & 1. Mitschke et al. (2018) \\
\hline & $\begin{array}{l}\text { Development of } \\
\text { standardized screening/ } \\
\text { assessment tools }\end{array}$ & $\begin{array}{l}\text { 1. Multiple } \\
\text { countries }\end{array}$ & 1. Not specified & 1. Review & $\begin{array}{l}\text { 1. Blight KJ., Ekblad S., } \\
\text { Lindencrona F., \& Shahnavaz } \\
\text { S. (2009) }\end{array}$ \\
\hline \multirow[t]{4}{*}{$\begin{array}{l}\text { Comprehensive } \\
\text { Mental } \\
\text { Healthcare }\end{array}$} & $\begin{array}{l}\text { Integration of social } \\
\text { services, family outreach, } \\
\text { school outreach, } \\
\text { community outreach, and/ } \\
\text { or service advocacy into } \\
\text { mental healthcare services }\end{array}$ & $\begin{array}{l}\text { 1. Southeast } \\
\text { Asia } \\
\text { 2. Southeast } \\
\text { Asia } \\
\text { 3. Asia } \\
\text { 4. Multiple } \\
\text { countries } \\
\text { 5. Multiple } \\
\text { countries } \\
\text { 6. Multiple } \\
\text { countries } \\
\text { 7. Multiple } \\
\text { countries } \\
\text { 8. Syria } \\
\text { 9. Multiple } \\
\text { countries }\end{array}$ & $\begin{array}{l}\text { 1. Not specified } \\
\text { 2. Adults } \\
\text { 3. Not specified } \\
\text { 4. Not specified } \\
\text { 5. Youth } \\
\text { 6. Children and } \\
\text { adolescents } \\
\text { 7. Children } \\
\text { 8. Not specified } \\
\text { 9. Adults }\end{array}$ & $\begin{array}{l}\text { 1. Case report } \\
\text { 2. Case report } \\
\text { 3. Review } \\
\text { 4. Review } \\
\text { 5. Review } \\
\text { 6. Case report } \\
\text { 7. Review } \\
\text { 8. Case report } \\
\text { 9. Primary } \\
\text { research }\end{array}$ & $\begin{array}{l}\text { 1. Murase K., Egawa J., \& } \\
\text { Tashima N. (1985) } \\
\text { 2. Allden K. (1998) } \\
\text { 3. Dhooper SS., \& Tran TV. } \\
\text { (1998) } \\
\text { 4. Watters C. (2001) } \\
\text { 5. Ellis HB., Miller AH., \& } \\
\text { Baldwin H., \& Abdi S. (2011) } \\
\text { 6. Rousseau C., Measham T., } \\
\text { \& Nadeau L. (2012) } \\
\text { 7. Pacione L., Measham T., \& } \\
\text { Rousseau C. (2013) } \\
\text { 8. Quosh C. (2013) } \\
\text { 9. Shannon PJ., Vinson GA., } \\
\text { Cook TL., \& Lennon E. } \\
\text { (2016) }\end{array}$ \\
\hline & $\begin{array}{l}\text { Multiethnic refugee } \\
\text { groups' involvement } \\
\text { in a community-based } \\
\text { participatory intervention } \\
\text { to develop culturally } \\
\text { competent training } \\
\text { with both western and } \\
\text { traditional healing } \\
\text { practices, build community } \\
\text { capacity to support mental } \\
\text { healthcare, and/or sustain } \\
\text { the intervention effect }\end{array}$ & $\begin{array}{l}\text { 1. Southeast } \\
\text { Asia } \\
\text { 2. Southeast } \\
\text { Asia } \\
\text { 3. Multiple } \\
\text { countries } \\
\text { 4. Multiple } \\
\text { countries } \\
\text { 5. Multiple } \\
\text { countries }\end{array}$ & $\begin{array}{l}\text { 1. Not specified } \\
\text { 2. Not specified } \\
\text { 3. Not specified } \\
\text { 4. Not specified } \\
\text { 5. Children }\end{array}$ & $\begin{array}{l}\text { 1. Case report } \\
\text { 2. Case report } \\
\text { 3. Case report } \\
\text { 4. Review } \\
\text { 5. Review }\end{array}$ & $\begin{array}{l}\text { 1. Lum RG. (1985) } \\
\text { 2. Murase K., Egawa J., \& } \\
\text { Tashima N. (1985) } \\
\text { 3. Eisenbruch M., de Jong J., } \\
\text { \& van de Put W. (2004) } \\
\text { 4. Weine SM. (2011) } \\
\text { 5. Pacione L., Measham T., \& } \\
\text { Rousseau C. (2013) }\end{array}$ \\
\hline & $\begin{array}{l}\text { Multidisciplinary } \\
\text { collaboration among } \\
\text { professionals in healthcare, } \\
\text { public health, social work, } \\
\text { advocacy, and legal and } \\
\text { political process }\end{array}$ & $\begin{array}{l}\text { 1. Somali } \\
\text { 2. Multiple } \\
\text { countries } \\
\text { 3. Multiple } \\
\text { countries }\end{array}$ & $\begin{array}{l}\text { 1. Adults } \\
\text { 2. Not specified } \\
\text { 3. Children }\end{array}$ & $\begin{array}{l}\text { 1. Primary } \\
\text { research } \\
\text { 2. Review } \\
\text { 3. Review }\end{array}$ & $\begin{array}{l}\text { 1. Palmer D. (2007) } \\
\text { 2. Blight KJ., Ekblad S., } \\
\text { Lindencrona F., \& Shahnavaz } \\
\text { S. (2009) } \\
\text { 3. Pacione L., Measham T., \& } \\
\text { Rousseau C. (2013) }\end{array}$ \\
\hline & $\begin{array}{l}\text { Comprehensive and } \\
\text { culturally appropriate } \\
\text { assessment with/without } \\
\text { family members included } \\
\text { in the evaluation }\end{array}$ & $\begin{array}{l}\text { 1. Southeast } \\
\text { Asia } \\
\text { 2. Multiple } \\
\text { countries }\end{array}$ & $\begin{array}{l}\text { 1. Adults } \\
\text { 2. Adults }\end{array}$ & $\begin{array}{l}\text { 1. Case report } \\
\text { 2. Case report }\end{array}$ & $\begin{array}{l}\text { 1. Allden K. (1998) } \\
\text { 2. d'Ardenne P., Capuzzo N., } \\
\text { Ruaro L., \& Priebe S. (2005) }\end{array}$ \\
\hline
\end{tabular}

were engaged in horizontal eye movement or other simulations [65], and it was implemented among Syria refugees using randomized controlled trial and was proven to be effective [65]. (2) Recognize traditional and/or culturally relevant mental health coping mechanism Besides seeking westernized mental healthcare, refugees in their host countries also practiced a variety of traditional and/or culturally relevant coping strategies which brought them comfort and safety [36,42,44,46,67-78]. Both religion and social support appeared to play an important role of alleviating refugees' psychiatric symptoms $[36,37,42,44,46,67-73]$. In one study, among 769 Tibetan refugee participants, practicing religion to cope with mental distress was most frequently used with $90 \%$ of the participants supporting this approach. About $77 \%$ of the participants would seek social support when they needed emotional support [69]. Refugees expressed their strong desire of talking to their families, peers, and friends when they were feeling down or troubled $[36,44,67,68,72,73]$. Participants in one study emphasized the significance of being part of a community peer group which consisted of members from "cooking, sewing, bible reading, women's, and senior groups [74]." The community peer group helped the participants get out of isolation, getting information about mental health and other health related issues, obtaining advice, and locating community services. They felt it was safe to share their experiences with each other within the group [74]. In addition, refugees also adopted many other coping strategies, including relaxation techniques (e.g., massage, meditation), dancing and listening to music, crying, exercising, reading and writing, self-analysis, and using Chinese or Southeast traditional medicine $[36,42,46,67,68,70-72,74-78]$. Several studies also stated the effectiveness of using acupuncture to treat symptoms of depression, anxiety and PTSD $[71,72,76]$. (3) Overcome existing barriers 
Ways identified by the current studies to address and overcome existing barriers mainly focused on education, training, and partnership building [26,42,51,72,73,79-96]. Educational interventions were developed to increase the awareness of the importance of mental wellbeing, knowledge of mental illnesses, and western models of treatment among refugee individuals, and their families and communities, reduce the stigma of having mental illnesses, and gain family and community support of mental healthcare especially with children and adolescents [26.42,51,72,80-85]. Family and community's involvement in these interventions were highly valued $[26,42,51,72$, $80,81]$. Cross-cultural psychiatric/competency training was also made available to mental healthcare providers and staff members for them to understand refugees populations' cultural understanding and interpretation of mental disorders and their traditional practice of treatment at home or in their countries of origin in order to improve the patient-doctor communication and the accuracy of assessment and diagnosis, integrate traditional care into western care, and meet refugees' mental health needs $[26,79,83,86,88,92]$. Refugee community members, including individual refugees, key informants, group leaders, leaders of cultural organization, religious leaders, and professional interpreters, were partnering with practitioners to exchange cross-cultural perspectives of health and serve as a liaison between their community and the medical society to building a trustworthy relationship $[51,73,74,85,89-92]$. Studies also suggested integrating ethnographical approach into mental healthcare practice for researchers and practitioners to gain a better understanding of refugee populations $[93,94]$; and integrating mental health services into existing services, such as school and primary care, to reduce stigma [51, 95, 96]. (4) Emphasize comprehensive/integrative mental healthcare models More and more comprehensive and integrative mental healthcare models have been proposed and implemented [26,42,51,55,82,84,85,87,91,97-112]. Effort was made to integrate social services, family outreach, school outreach, community outreach, and/or service advocacy into mental healthcare and facilitate multidisciplinary collaboration among professionals in healthcare, public health, social work, advocacy, and legal and political process $[26,42,51,55,82,85,87,91,97-99]$. One study illustrated the factors contributed to the successful mental health referrals among multiethnic refugee groups. Mental health providers were embedded in health or non-health settings to support an interdiciplinary coordinated care for refugees. An employment counselor could refer a refugee client internally to an onsite mental health provider in the same agency [85]. Providers were willing to give a refugee client with an initial assessment and/or follow-up services at the client home in order to provide responsive care and accommodate the client's inconvenience of being physically present at the clinic. Sometimes, transportation was also arranged and offered by an agency to pick up a refugee client and send him/her to their providers. Multidisciplinary care was further expanded to meet refugee clients' non-clinical needs, including helping them navigate additional services and resources, advocating for them, and assisting them in completing paperwork for medical assistance [85]. Comprehensive assessment, including psychiatric and medical history, trauma experiences, mental health and general health status, cognitive assessment, social function, and quality of life, were also suggested along with involving family members in the evaluation to have a better understanding of a refugee client for diagnosis and treatment if possible especially given the significance of family impact on an individual refugee [82,84]. Interventions using community-based participatory approach have a long-lasting impact on improving refugee populations' mental health. Community members took the ownership to develop their own culturally and linguistically competent and tailored mental health interventions which supported community capacity building, individual community members' empowerment, and sustainability of the effects of the interventions [91,98,100-112].

\section{Discussion}

In 2015, United Nations High Commissioner for Refugees et al. published a guide for mental health and psychosocial support for refugees, asylum seekers and migrants on the move in Europe, included 12 principles (1) "treat all people with dignity and respect and support self-reliance;" (2) "respond to people in distress in a humane and supportive way;" (3) "provide information about services, supports and legal rights and obligations;" (4) "provide relevant psycho-education and use appropriate language;" (5) "prioritize protection and psychosocial support for children, in particular children who are separated, unaccompanied and with special needs;" (6) "strengthen family support;" (7) "identify and protect persons with specific needs;" (8) "make interventions culturally relevant and ensure adequate interpretation;" (9) "provide treatment for people with severe mental disorders;" (10) "do not start psychotherapeutic treatments that need follow up when follow up is unlikely to be possible;" (11) "monitoring and managing wellbeing of staff and volunteers;" and (12) "do not work in isolation: coordinate and cooperate with others [103]." Recommendations made by the World Psychiatric Association (WPA) and European Psychiatric Association (EPA) for immigrants and refugees were consistent with these 12 principles [104,105]. Specifically, both WPA and EPA emphasized the critical needs of preparing a culturally competent mental health workforce, and a regular assessment of local refugees and immigrants' mental health needs, and availability and accessibility of the services $[103,106]$. It was suggested that cultural brokers or liaisons be hired and be part of the services, and cultural competent training be made mandatory for service providers and clinicians and be integrated into curriculum [104,105].

As one of the largest refugee resettlement countries, based on the review, U.S. has experienced extremely similar challenges to the ones existed in many other western countries, including UK, Canada, and Australia. U.S. along with other western countries has been working on improving the availability, accessibility, and credibility of mental health services among refugees in their countries. The themes of the current endeavor in practice emerged from this systematic review to address refugees' mental healthcare needs are clearly aligned with the proposed principles and recommendations, which included (1) adopting westernized mental health treatment that has been proven to be effective among refugees, such as different forms of trauma-focused therapies; (2) recognizing traditional and/or culturally relevant mental health coping mechanism, such as religion, social support, and traditional healing practice, and integrate them into western treatment models; (3) acknowledging and removing existing cultural, linguistic, and system barriers for refugee populations to effectively access and utilize the mental health services through educating and training both refugees and practitioners to enhance the practitioners' understanding of cultural interpretation of mental illnesses, and their accuracy of assessment and diagnosis and provision of cultural responsive care, and improve refugees' awareness of the importance of mental wellbeing and their acceptance of westernized treatment; as well as through establishing a trusting and long-term partnership between refugees and their families and communities, and practitioners; and (4) emphasizing comprehensive/integrative mental healthcare models by focusing on multidisciplinary collaboration of services and professionals in social work, public health, healthcare, advocacy, and legal and political process, and community-based participatory mental health interventions. However, more future endeavor needs to be devoted to systematically train the healthcare personnel and prepare a culturally competent workforce in order to assure the implementation of the policies and guiding principles and recommendations. The guidelines on mental health training of primary healthcare providers for traumaexposed populations in conflict-affected countries sponsored by the International Society for Traumatic Stress Studies and Research 
ANd Development Cooperation (RAND) suggested that effective and quality training should emphasize multidisciplinary collaboration and participation in assessing training needs, planning training activities, designing cultural appropriate training curriculum, advocating for a policy change with mobilizing family and community's involvement and using human rights as a framework; both the design and implementation of training should consider the cultural and socioecological contributions to mental health needs and understand the perceived mental health service needs in the community and the training needs among service providers; the training curriculum should be evidence-based with including both knowledge and skills for mental health, understanding of interaction between physical health and mental health, culturally appropriate treatments, skills for assessment and communication, awareness of confidentiality and trust, and awareness of community resources; and providing science and context-informed training with a proper selection of trainers and trainees, and training models and a careful plan for monitoring and evaluation of the training [106].

Although the primary focus of the current systematic review was to explore the existing barriers to the utilization of mental health services among refugees and potential solutions to the effectiveness of addressing their mental health needs, based on the findings, in addition to the training needs, more research/evaluation needs to be developed to further demonstrate the effectiveness of training curriculum, community-based mental health interventions, integrative care, traditional healing practice, as well as psychotherapies among different refugee populations with better study designs and larger sample sizes in order to provide solid evidence and guide evidencebased practice $[107,108]$. Using biomedical model and epidemiology, research is needed to examine the psychopathology and demonstrate the cross-cultural credibility of clinical and non-clinical treatment along with more qualitative studies to explore the conceptualization of mental illnesses and mental health among refugees [104,107].

While measures have been taken to address the barriers of the underutilization of mental healthcare among refugees in their host countries and address their mental health needs, the needs remain unmet especially given the significantly increasing number of refugees worldwide and the long-term impact of mental illnesses on refugees' mental wellbeing and productivity, as well as on global and individual country's burden of disease [1, 2, 18-23]. Refugee populations' mental health needs are tremendous and ongoing. A report on an evaluation of the primary healthcare needs of refugees in south east metropolitan Melbourne, Australia in 2011 identified a strikingly high use of community-based mental health services among Burmese refugees, and stated that although local specialized mental healthcare particularly intended to address the high prevalence and incidence of mental illnesses among local refugees, a long patient waiting list and an apparent inadequacy of refugee-specialized psychologists and psychiatrists raised serious concerns about the local capacity of fully satisfying the refugees' mental health needs [109]. In 2003, World Health Organization's Project Atlas reported baseline information on 89 countries' budget and financing on mental health services. About $32 \%$ of its 191 member countries did not have a specified budget for mental health, and $36 \%$ of 89 countries had a less than $1 \%$ of total health budget on mental healthcare. Although low-income countries tended to spend less money on mental healthcare, many highincome countries spent less than $5 \%$ of their health budget on mental health [110]. Countries spent more than $5 \%$ were also challenged by institutional and organizational barriers, lack of accountability, and other issues in terms of service delivery [110]. There was a significant imbalance between disease burden and resource allocation [110]. Findings suggested a serious consideration of budget increase on mental health services to increase service, training, and research opportunities, and emphasized more operational research studies should be done to examine the expenditure of mental healthcare among different populations, optimize the allocation of current resources, and provide policy development, implementation and evaluation, and service planning with empirical data and guidance [110], which were consistent with other study findings [111,112].

\section{Conclusions}

Mental health services have been historically under-utilized among refugee populations in their resettlement countries. Resolutions to current challenges faced by many western countries associated with the underutilization of services have focused on identifying and adopting effective trauma-centered therapies, removing existing practical barriers within both refugee communities and western mental healthcare systems, recognizing traditional and/or culturally relevant coping strategies, and delivering multifaceted multidisciplinary community-based participatory mental health interventions. More future endeavor should be dedicated to the areas of systematically integrating planned cultural competency training and education into existing curriculum, strengthening the quality of mental healthcare research with refugees, and advocating for funding support for mental healthcare services, training, and research.

\section{References}

1. United Nation Refugee Agency (2018).

2. U.S. Department of State (2018).

3. Alemi Q, James S, Cruz R, Zepeda V, Racadio M et al. (2014) Psychological distress in Afghan refugees: A mixed-method systematic review. J Iimmigr Minor health 16: 1247-1261.

4. Fazel M, Wheeler J, Danesh J (2005) Prevalence of serious mental disorder in 7000 refugees resettled in western countries: a systematic review. The Lancet 365: 1309-1314.

5. Lindert J, Ehrenstein VOS, Priebe S, Mielck A, Brähler E et al. (2009) Depression and anxiety in labor migrants and refugees-a systematic review and meta-analysis. Soc Sci Med 69: 246-257.

6. Mills E, Singh S, Roach B, Chong S (2008) Prevalence of mental disorders and torture among Bhutanese refugees in Nepal: a systemic review and its policy implications. Med Confl Surviv 24: 5-15.

7. Steel Z, Chey T, Silove D, Marnane C, Bryant RA et al. (2009) Association of torture and other potentially traumatic events with mental health outcomes among populations exposed to mass conflict and displacement: a systematic review and metaanalysis. JAMA 302: 537-549.

8. Taylor EM, Yanni EA, Pezzi C, Guterbock M, Rothney E et al. (2014) Physical and mental health status of Iraqi refugees resettled in the United States. J Immigr Minor Health 16: 11301137.

9. Cardozo BL, Bilukha OO, Gotway CA, Wolfe MI, Gerber ML et al. (2005) Report from the CDC: Mental health of women in postwar Afghanistan. J Womens Health 14: 285-293.

10. Collier AF, Munger M, Moua YK (2012) Hmong mental health needs assessment: A community-based partnership in a small mid-western community. Am J Community Psychol 49: 73-86.

11. Gerritsen AA, Bramsen I, Devillé W, Van Willigen LH, Hovens JE et al. (2006) Physical and mental health of Afghan, Iranian and Somali asylum seekers and refugees living in the Netherlands. Soc Psychiatry Psychiatr Epidemiol 41: 18-26.

12. Marshall GN, Schell TL, Elliott MN, Berthold SM, Chun CA et al. (2005) Mental health of Cambodian refugees 2 decades after resettlement in the United States. JAMA 294: 571-9.

13. Mollica RF, Caridad KR, Massagli MP (2007) Longitudinal study of posttraumatic stress disorder, depression, and changes in traumatic memories over time in Bosnian refugees. J Nerv Ment Dis 195: 572-9. 
14. Attanayake V, McKay R, Joffres M, Singh S, Burkle Jr F et al. (2009) Prevalence of mental disorders among children exposed to war: a systematic review of 7,920 children. Med Confl Surviv 25: 4-19.

15. Ellis BH, MacDonald HZ, Lincoln AK, Cabral HJ (2008) Mental health of Somali adolescent refugees: the role of trauma, stress, and perceived discrimination. J Consult Clin Psychol 76: 184.

16. Fazel M, Stein A (2003) Mental health of refugee children: comparative study. BMJ 327: 134.

17. Huemer J, Karnik NS, Voelkl-Kernstock S, Granditsch E, Dervic K et al. (2009) Mental health issues in unaccompanied refugee minors. Child Adolesc Psychiatry Ment Health 3: 13.

18. McCrone P, Bhui K, Craig T, Mohamud S, Warfa N et al. (2005) Mental health needs, service use and costs among Somali refugees in the UK. Acta Psychiatr Scand 111: 351-357.

19. Fenta H, Hyman I, Noh S (2006) Mental health service utilization by Ethiopian immigrants and refugees in Toronto. $\mathrm{J}$ Nerv Ment Dis 194: 925-934.

20. Bean T, Eurelings-Bontekoe E, Mooijaart A, Spinhoven P (2006) Factors associated with mental health service need and utilization among unaccompanied refugee adolescents. Adm Policy Ment Health 33: 342-355.

21. Fenta H, Hyman I, Noh S (2007) Health service utilization by Ethiopian immigrants and refugees in Toronto. J Immigr Minor Health 9: 349-357.

22. Nosè M, Turrini G, Barbui C (2015) Access to mental health services and psychotropic drug use in refugees and asylum seekers hosted in high-income countries. Epidemiol Psychiatr Sci 24: 379-381.

23. Gaebel W, Falkai P, Sartorius N, Zielasek J (2016) Mental healthcare for refugees. Psychiatrie 13: 61-64.

24. Gong-Guy E, Cravens RB, Patterson TE. (1991) Clinical issues in mental health service delivery to refugees. Am Psychol 46: 642-648.

25. Gold SJ (1992) Mental health and illness in Vietnamese refugees. West J Med 157: 290-294.

26. Dhooper SS, Tran TV (1998) Understanding and responding to the health and mental health needs of Asian refugees. Soc Work Health Care 27: 65-82.

27. Blair RG (2001) Mental health needs among Cambodian refugees in Utah. Int Soc Work 44: 179-196.

28. Scuglik DL, Alarcón RD, Lapeyre III AC, Williams MD, Logan KM (2007) When the poetry no longer rhymes: Mental health issues among Somali immigrants in the USA. Transcult Psychiatry 44: 581-595.

29. Pavlish CL, Noor S, Brandt J (2010) Somali immigrant women and the American health care system: discordant beliefs, divergent expectations, and silent worries. Soc Sci Med 71: 353-361.

30. Wagner J, Burke G, Kuoch T, Scully M, Armeli S et al. (2013) Trauma, healthcare access, and health outcomes among Southeast Asian refugees in Connecticut. J Immigr Minor Health 15: 1065-1072.

31. Morris MD, Popper ST, Rodwell TC, Brodine SK, Brouwer KC et al. (2009) Healthcare barriers of refugees post-resettlement. J Community health 34: 529-538.

32. Lee HY, Lytle K, Yang PN, Lum T (2010) Mental health literacy in Hmong and Cambodian elderly refugees: A barrier to understanding, recognizing, and responding to depression. Int $\mathrm{J}$ Aging Hum Dev 71: 323-344.
33. Shannon P, O'Dougherty M (2012) Mehta E. Refugees' perspectives on barriers to communication about trauma histories in primary care. Ment Health Fam Med 9: 47-55.

34. Shannon PJ, Wieling E, Simmelink-McCleary J, Becher E (2015) Beyond stigma: Barriers to discussing mental health in refugee populations. J Loss and Trauma 20: 281-296.

35. Nguyen SD (1985) Mental Health Services for Refugees and Immigrants in Canada in Owan TC. Southeast Asian Mental Health: Treatment, Prevention, Services, Training and Research. ERIC.

36. Carroll JK (2004) Murug, Waali, and Gini: expressions of distress in refugees from Somalia. Prim Care Companion J Clin Psychiatry 6: 119-125.

37. Bettmann JE, Penney D, Clarkson Freeman P, Lecy N (2015) Somali refugees' perceptions of mental illness. Soc Work Health Care 54: 738-757.

38. Frye BA, McGill D (1993) Cambodian refugee adolescents: Cultural factors and mental health nursing. J Child Adolesc Psychiatr Nurs 6: 24-31.

39. Murphy D, Ndegwa D, Kanani A, Rojas-Jaimes C, Webster A et al. (2002) Mental health of refugees in inner-London. The Psychiatrist 26: 222-224.

40. Misra T, Connolly AM, Majeed A (2006) Addressing mental health needs of asylum seekers and refugees in a London Borough: epidemiological and user perspectives. Prim Health Care Res Dev 7: 241-248.

41. Palmer D (2006) Imperfect prescription: mental health perceptions, experiences and challenges faced by the Somali community in the London Borough of Camden and service responses to them. Primary Care Ment Health 4: 45-56.

42. Palmer D (2007) Caught between inequality and stigma: the impact of psychosocial factors and stigma on the mental health of Somali forced migrants in the London Borough of Camden. Diversity Health Soc Care 4: 177-191.

43. Franks W, Gawn N, Bowden G (2007) Barriers to access to mental health services for migrant workers, refugees and asylum seekers. J Public Ment Health 6: 33-41.

44. De Anstiss H, Ziaian T (2010) Mental health help-seeking and refugee adolescents: Qualitative findings from a mixed-methods investigation. Australian Psychol 45: 29-37.

45. Majumder P, O'Reilly M, Karim K, Vostanis P (2015) 'This doctor, I not trust him, I'm not safe': The perceptions of mental health and services by unaccompanied refugee adolescents. Int J Soc Psychiatry 61: 129-136.

46. Slewa-Younan S, Mond J, Bussion E, Mohammad Y, Guajardo MG et al. (2014) Mental health literacy of resettled Iraqi refugees in Australia: Knowledge about posttraumatic stress disorder and beliefs about helpfulness of interventions. BMC psychiatry 14: 320-327.

47. De Anstiss H, Ziaian T, Procter N, Warland J, Baghurst P et al. (2009) Help-seeking for mental health problems in young refugees: A review of the literature with implications for policy, practice, and research. Transcult Psychiatry 46: 584-607.

48. Savic M, Chur-Hansen A, Mahmood MA, Moore VM (2016) 'We don't have to go and see a special person to solve this problem': Trauma, mental health beliefs and processes for addressing 'mental health issues' among Sudanese refugees in Australia. Int J Soc Psychiatry 62: 76-83.

49. Beiser M, Simich L, Pandalangat N (2003) Community in Distress: Mental Health Needs and Help-seekingin the Tamil Community in Toronto. Int Migra 41: 233-245. 
50. Donnelly TT, Hwang JJ, Este D, Ewashen C, Adair C et al. (2011) If I was going to kill myself, I wouldn't be calling you. I am asking for help: Challenges influencing immigrant and refugee women's mental health. Issues Ment Health Nur 32: 279-290

51. Ellis HB, Miller AB, Baldwin H, Abdi S (2011) New directions in refugee youth mental health services: Overcoming barriers to engagement. J Child Adolesc Trauma 4: 69-85.

52. Warfa N, Bhui K, Craig T, Curtis S, Mohamud S (2006) Thornicroft G. Post-migration geographical mobility, mental health and health service utilisation among Somali refugees in the UK: A qualitative study. Health Place12: 503-515.

53. Ward K, Palmer D (2005) Mapping the provision of mental health services for asylum seekers and refugees in London: a report. London: Commission for Public Patient Involvement in Health.

54. Feldman R (2006) Primary health care for refugees and asylum seekers: a review of the literature and a framework for services. Public Health 120: 809-816.

55. Watters C (2001) Emerging paradigms in the mental health care of refugees. Soc Sci Med 52: 1709-1718.

56. Murray KE, Davidson GR, Schweitzer RD (2010) Review of refugee mental health interventions following resettlement: best practices and recommendations. Am J Orthopsychiatry 80: 576585.

57. Crumlish N, O'rourke K (2010) A systematic review of treatments for post-traumatic stress disorder among refugees and asylum-seekers. J Nerv Ment Dis 198: 237-251.

58. Palic S, Elklit A (2011) Psychosocial treatment of posttraumatic stress disorder in adult refugees: A systematic review of prospective treatment outcome studies and a critique. J Affect Disord 131: 8-23.

59. Slobodin O, de Jong JT (2015) Mental health interventions for traumatized asylum seekers and refugees: What do we know about their efficacy? Int J Soc Psychiatry 61: 17-26.

60. Stenmark H, Catani C, Neuner F, Elbert T, Holen A et al. (2013) Treating PTSD in refugees and asylum seekers within the general health care system. A randomized controlled multicenter study. Behav Res Ther 51: 641-647.

61. Schulz PM, Huber LC, Resick PA (2006) Practical adaptations of cognitive processing therapy with Bosnian refugees: Implications for adapting practice to a multicultural clientele. Cogn Behav Pract 13: 310-321.

62. Schulz PM, Resick PA, Huber LC, Griffin MG (2006) The effectiveness of cognitive processing therapy for PTSD with refugees in a community setting. Cogn Behav Pract 13: 322331.

63. Nickerson A, Bryant RA, Silove D, Steel Z (2011) A critical review of psychological treatments of posttraumatic stress disorder in refugees. Clin Psychol Rev 31: 399-417.

64. Arntz A, Sofi D, van Breukelen G (2013) Imagery Rescripting as treatment for complicated PTSD in refugees: a multiple baseline case series study. Behav Res Ther 51: 274-283.

65. Acarturk C, Konuk E, Cetinkaya M, Senay I, Sijbrandij M et al. (2015) EMDR for Syrian refugees with posttraumatic stress disorder symptoms: Results of a pilot randomized controlled trial. Eur J Psychotraumatol 6: 27414.

66. Weine SM, Kuc G, Dzudza E, Razzano L, Pavkovic I et al. (2001) PTSD among Bosnian refugees: A survey of providers' knowledge, attitudes and service patterns. Community Ment Health J 37: 261-271.
67. Whittaker S, Hardy G, Lewis K, Buchan L (2005) An exploration of psychological well-being with young Somali refugee and asylum-seekerwomen.ClinChildPsycholPsychiatry 10:177-196.

68. Khawaja NG, White KM, Schweitzer R, Greenslade J (2008) Difficulties and coping strategies of Sudanese refugees: A qualitative approach. Transcult. Psychiatry 45: 489-512.

69. Sachs E, Rosenfeld B, Lhewa D, Rasmussen A, Keller A et al. (2008) Entering exile: Trauma, mental health, and coping among Tibetan refugees arriving in Dharamsala, India. J Trauma Stress 21: 199-208.

70. MacDuff S, Grodin MA, Gardiner P (2011) The use of complementary and alternative medicine among refugees: A systematic review. J Immigr Minor Health 13: 585-599.

71. Mckenna Longacre MM, Silver-Highfield E, Lama P, Grodin MA et al. (2012) Complementary and alternative medicine in the treatment of refugees and survivors of torture: A review and proposal for action. Torture 22: 38-57.

72. Guerin B, Guerin PB, Diiriye RO, Yates S (2004) Somali conceptions and expectations concerning mental health: Some guidelines for mental health professionals. NZ J Psychol 33: 59-66.

73. Mitschke DB, Praetorius RT, Kelly DR, Small E, Kim YK et al. (2018) Listening to refugees: How traditional mental health interventions may miss the mark. Int. Soc. Work 60: 588-600.

74. Behnia B (2003) Refugees' convoy of social support: Community peer groups and mental health services. Int J Ment Health 32: 6-19.

75. Chung RC, Lin KM (1994) Help-seeking behavior among Southeast Asian refugees. J Community Psychol 22: 109-120.

76. Pease M, Sollom R, Wayne P (2009) Acupuncture for refugees with posttraumatic stress disorder: initial experiences establishing a community clinic. Explore: J Sci Healing 5: 5154.

77. Gustafson MB. (1989) Western voodoo: Providing mental health care to Haitian refugees. J Psychosoc Nurs Ment Health Serv 27: 22-25.

78. Coker EM (2004) "Traveling pains": Embodied metaphors of suffering among Southern Sudanese refugees in Cairo. Cult Med Psychiatry 28: 15-39.

79. True PH (1985) An Indochinese Mental Health Service Model in San Franciscoin in Owan TC. Southeast Asian Mental Health: Treatment, Prevention, Services, Training and Research. ERIC.

80. Weine S, Kulauzovic Y, Klebic A, Besic S, Mujagic A et al. (2008) Evaluating a multiple-family group access intervention for refugees with PTSD. J Marital Fam Ther 34: 149-164.

81. Nazzal KH, Forghany M, Geevarughese MC, Mahmoodi V, Wong J (2014) An innovative community-oriented approach to prevention and early intervention with refugees in the United States. Psychol Sev 11: 477-485.

82. Allden K (1998) The Indochinese Psychiatry Clinic: trauma and refugee mental health treatment in the 1990s. J Ambul Care Manage 21: 30-38.

83. Chung RC (2001) Psychosocial adjustment of Cambodian refugee women: Implications for mental health counseling. J Ment Health Couns 23:115-126.

84. Ardenne DP, Capuzzo N, Ruaro L, Priebe S (2005) One size fits all? Cultural sensitivity in a psychological service for traumatised refugees. Divers Equal Health Care 2: 29-36.

85. Shannon PJ, Vinson GA, Cook TL, Lennon E (2016) Characteristics of successful and unsuccessful mental health referrals of refugees. Adm Policy Ment Health 43: 555-568. 
86. Burnett A, Gebremikael L (2005) Expanding the primary mental health team for refugees and asylum seekers. Primary Care Ment Health 3:77-80.

87. Johansson Blight K, Ekblad S, Lindencrona F, Shahnavaz S (2009) Promoting mental health and preventing mental disorder among refugees in Western countries. Int J Ment Health Promot 11: 32-44.

88. Bäärnhielm S, Edlund AS, Ioannou M, Dahlin M (2014) Approaching the vulnerability of refugees: evaluation of crosscultural psychiatric training of staff in mental health care and refugee reception in Sweden. BMC Med Educ 14: 207-217.

89. Kirmayer LJ, Groleau D, Guzder J, Blake C, Jarvis E (2003) Cultural consultation: A model of mental health service for multicultural societies. Can J Psychiatry 48: 145-153.

90. Shannon PJ (2014) Refugees' advice to physicians: how to ask about mental health. J Fam Pract 31:462-466.

91. Murase K, Egawa J, Tashima N (1985) Alternative mental health service models in Asian/Pacific communities in Owan TC. Southeast Asian Mental Health: Treatment, Prevention, Services, Training and Research. ERIC.

92. Crosby SS (2013) Primary care management of non-Englishspeaking refugees who have experienced trauma: a clinical review. JAMA 310: 519-528.

93. Gozdziak EM (2004) Training refugee mental health providers: Ethnography as a bridge to multicultural practice. Hum Organ 63: 203-210.

94. Kohrt BA, Maharjan SM, Timsina D, Griffith JL (2012) Applying Nepali ethnopsychology to psychotherapy for the treatment of mental illness and prevention of suicide among Bhutanese refugees. Ann Anthropol Pract 36: 88-112.

95. Fazel M, Doll H, Stein A (2009) A school-based mental health intervention for refugee children: An exploratory study. Clin Child Psychol Psychiatry 14: 297-309.

96. White CC, Solid CA, Hodges JS, Boehm DH (2015) Does integrated care affect healthcare utilization in multi-problem refugees? J Immigr Minor Health 17: 1444-1450.

97. Rousseau C, Measham T, Nadeau L (2013) Addressing trauma in collaborative mental health care for refugee children. Clin Child Psychol Psychiatry 18:121-36.

98. Pacione L, Measham T, Rousseau C (2013) Refugee children: Mental health and effective interventions. Current psychiatry reports 15: 341-357.

99. Quosh C (2013) Mental health, forced displacement and recovery: integrated mental health and psychosocial support for urban refugees in Syria. Intervention 11: 295-320.

100. Lum RG (1985) A community-based mental health services to Southeast Asian refugees in Asian/Pacific communities in Owan TC. Southeast Asian Mental Health: Treatment, Prevention, Services, Training and Research. ERIC.

101. Eisenbruch M, de Jong JT, van de Put W (2004) Bringing order out of chaos: A culturally competent approach to managing the problems of refugees and victims of organized violence. $\mathrm{J}$ Trauma Stress 17: 123-131.

102. Weine SM (2011) Developing preventive mental health interventions for refugee families in resettlement. Fam Process 50: $410-430$

103. Ventevogel P, Schinina G, Strang A, Gagliato M, Hansen LJ et al. (2015) Mental health and Psychosocial Support for Refugees, Asylum Seekers and Migrants on the move in Europe: a MultiAgency Guidance note
104. V Bhugra D, Gupta S, Bhui K, Craig TO, Dogra N (2011) WPA guidance on mental health and mental health care in migrants. World Psychiatry 10: 2-10.

105. Bhugra D, Gupta S, Schouler-Ocak M, Graeff-Calliess I, Deakin NA et al. (2014) EPA guidance mental health care of migrants. Eur Psychiatry 29: 107-115.

106. Eisenman D, Weine S, Green B, Jong JD, Rayburn N et al. (2006) The ISTSS/Rand Guidelines on Mental Health Training of Primary Healthcare Providers for Trauma-Exposed Populations in Conflict-Affected Countries. J Trauma Stress 19: 5-17.

107. Tempany M (2009) What research tells us about the mental health and psychosocial wellbeing of Sudanese refugees: A literature review. Transcult Psychiatry. 46: 300-315.

108. Crumlish N, O'rourke K (2010) A systematic review of treatments for post-traumatic stress disorder among refugees and asylum-seekers. J Nerv Ment Dis 198: 237-251.

109. Cheng IH, Russell G, Bailes M, Block A (2011) An evaluation of the primary healthcare needs of refugees in South East Metropolitan Melbourne.

110. Saxena S, Sharan P, Saraceno B (2003) Budget and financing of mental health services: baseline information on 89 countries from WHO's project atlas. J Ment Health Policy Econ 6: 135143.

111. Reitmanova S, Gustafson DL (2009) Mental health needs of visible minority immigrants in a small urban center: Recommendations for policy makers and service providers. $\mathrm{J}$ Immigr Minor Health 11: 46-56.

112. Boehnlein JK (1987) A review of mental health services for refugees between 1975 and 1985 and a proposal for future services. Psychiatr Serv 38: 764-768. 